\title{
A Comparative Analysis of Energy Costing Methodologies
}

Appendix: Report on Levelized Busbar-
Costing Workshop Held at
MITRE/Metrek, June $29 \& 30,1978$

J. G. Leigh

February 1979

Contract Sponsor: DOE-DGE

Contract No.: EG-77-C-01-4014

Project No.: $1526 \mathrm{~V}$

Dept.: W-52
The MITRE Corporation

Metrek Division 1820 Dolley Madison Boulevard McLean, Virginia 22102

MITRE Technical Report MTR-7689 


\section{DISCLAIMER}

This report was prepared as an account of work sponsored by an agency of the United States Government. Neither the United States Government nor any agency Thereof, nor any of their employees, makes any warranty, express or implied, or assumes any legal liability or responsibility for the accuracy, completeness, or usefulness of any information, apparatus, product, or process disclosed, or represents that its use would not infringe privately owned rights. Reference herein to any specific commercial product, process, or service by trade name, trademark, manufacturer, or otherwise does not necessarily constitute or imply its endorsement, recommendation, or favoring by the United States Government or any agency thereof. The views and opinions of authors expressed herein do not necessarily state or reflect those of the United States Government or any agency thereof. 


\section{DISCLAIMER}

Portions of this document may be illegible in electronic image products. Images are produced from the best available original document. 


\begin{abstract}
This document describes the proceedings of a workshop on leve1ized busbar costing methodologies which was held at MITRE/Metrek on June 29 and 30,1978 . The workshop was sponsored by the Division of Geothermal Energy within the Department of Energy and particular emphasis was placed on consideration of geothermal energy sources. The objective of the workshop was to determine whether a consensus could be developed regarding the most appropriate methodologies and assumptions for levelized energy costing. The workshop was attended by representatives from energy resource, utility and engineering design companies, and by representatives of the Division of Geotherma1 Energy and R\&D contractors for this Division.

It was found that year-by-year calculations in current dollars were generally preferred, using either Discounted Cash Flow or Revenue Requirements methods. No consensus emerged on choice of discount rate or finanacial parameters such as debt/equity ratio, and tax credit carry forward/carry back provisions. It was felt that englneering aspects deserve close attention.
\end{abstract}



$\begin{array}{lll}1.0 & \text { INTRODUCTION } & 1\end{array}$

1.1 Background

1.2 Organization of this Report

1

2

2.0. WORKSHOP DISCUSSIONS 4

2.1 Choice of Methodological Approach 4

2.2 Treatment of Inflation 6

2.3 Choice of Discount Rate 7

2.4 Depreciation Method 8

2.5 Other Financial Assumptions and Parameters 8

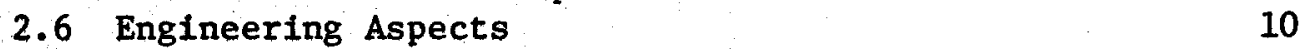

3.0 CONCLUSIONS AND RECOMMENDATIONS 12

ATTACHMENT A - LIST OF ATTENDEES 13

ATTACHMENT B - PAPERS PRESENTED AT THE WORKSHOP 17

ATTACHMENT C - AN ANALYSIS OF RR AND DCF APPROACES $\quad 69$

DISTRIBUTION LIST — $\quad-75$ 


\subsection{INTRODUCTION}

\subsection{Background}

In 1977, the Division of Geothermal Energy (DGE) in the Department of Energy asked MITRE/Metrek to examine different methodologies which were in use for projecting expected costs of electricity from geothermal sources. This request was motivated by the discovery that radically different electricity costs were being projected by different organizations for the same geothermal and conventional (1.e., coal and nuclear) energy sources. This finding generated some concern because reliable cost projections are fundamental to the proper direction and management of DGE's programs in fiscal incentives, RD\&D and loan guaranties.

During this work, MITRE/Metrek compiled and analysed the financlal methodologies and assumptions used by 14 organizations active In the geothermal field. These organizations included energy resource compantes, electric utilities, engineering companies, and R\&D contractors for DGE. The detailed results of this investigation have been published.* Here, it is sufficient to note that it rapldly became apparent that differences in financial methodologies and assumptions could lead to projected electricity costs differing by a factor of 2 for Identical physical geothermal resources and generating plants.

* See the main volume of this report. 
The preliminary results of this work were circulated to DGE and the participating organizations during the early part of 1978. Because of the response generated, it was decided to hold a workshop for representatives from these organizations, in order to obtain the benefit of face-to-face interchange and discussion. The workshop objective was to, if possible, reconcile differences between approachs and to develop recommendations to DGE for preferred methods and assumptions. The workshop was held at MITRE/Metrek on June 29 and 30, 1978. A 1ist of attendees is given in Attachment A.

\subsection{Organization of This Report}

The maln body of this report (Section 2) consists of synopses of discussions which took place during the workshop. These have been organized into topics which represent areas where significant choices have to be made in developing a financlal methodology. The topics are:

- Cholce of Methodological Approach

- Treatment of Inflation

- Selection and Use of Discount Rate

- Choice of Depreciation Method

- Other Financial Assumptions and Parameters

- Engineering Aspects

In addition to summarizing, as far as possible, the differing views expressed by workshop particlpants, MITRE/Metrek has attempted to give the consensus of the group in cases where this was felt to 
exist. It must be emphasized that this material represents MITRE/ Metrek's Impressions only, and has not been formally reviewed by the other workshop participants, However, every effort has been made to present differing opinions fully and fairly.

Section 3 of this report contains a list of recommendations which emerged from the workshop, and, finally, Attachments A and B contain a list of participants and copies of materials presented, respectively. 


\subsection{WORKSHOP DISCUSSIONS}

For ease of presentation, the wide-ranging discussions which took place during the workshop sessions have been summarized under a number of separate headings covering the major topics of importance. These synopses are presented in this section. Conclusions and recommendations are summarized in Section 3.

\subsection{Choice of Methodological Approach}

There are several possibilities for the cholce of the basic methodological approach or structure of the financial model. These include the use of year-by-year calculations versus the use of closedform equations, and the use of discounted cash flow (DCF) methods versus the use of revenue requirements (RR) methods.

Year-by-year calculations, as the name implies, involve the successive calculation of expenditures (capital, O\&M costs, etc.), depreciation, taxes, etc. for each year of the project (including pre-start-up investment). Levelization of the cash flow or cost stream is carried out to arrive at a levelized cost. In contrast, closed-form methods employ algebraic equations to express the present values of all costs, revenues, taxes, etc., relying upon the substitution of particular values in the equations to evaluate specific cases.

Year-by-year calculations are more flexible and can be more detalled than closed-form methods, and they can capture procedures which would be cumbersome to express mathematically. An example is 
the common practice of switching from double declining balance to straight line depreciation half way through the project life. This is easy to do in year-by-year calculations, but awkward to handle as a closed form equation. Year-by-year methods also generally make more Information available. However, closed-form methods generally require less computation. It was the feeling of the participants that yearby-year methods are preferable.

DCF methods generally compute the present value (in the start-up year) of the cash flow associated with the projects. It is usual to choose a price ( $1 . e .$, levelized cost) which makes the present value of the cash flow zero. RR methods, on the other hand, work from a knowledge of the expected costs (including "profit" or returns to equity), and then derive the revenue which would cover these costs. Thus the profits are explicitly bullt in, while in DCF methods profits are implicitly present in the choice of discount rate.

There was little agreement as to whether DCF or RR methods were preferable. It appears that utility companies tend to use $R R$, while energy resource companies tend to use DCF. It is probably true that DCF represents a more sophisticated approach to the problem, since this technique was historically developed later than RR. It may well be that the choice may depend on the organization being modelled (to reflect the perceptions of that organization). However, this is hardly appropriate for a national effort such as DGE's. It has been shown that, if all parameters including espectally discount rate, are 
correctly chosen, then DCF and RR lead to equivalent results*. Failing this, a possible compromise would be to perform RR calculations, but also give the internal rate of return to which these lead. Under the circumstances, MITRE/Metrek feels that no consensus emerged from the workshop on this point.

On further methodological questions, the participants felt very clearly that the development of geothermal resources would be split between field developers and utility-type companies, and the view was expressed that different financlal approaches might be appropriate for these organizations. In particular, DCF might be used for the field developer, and RR for the utility.

\subsection{Treatment of Inflation}

The participants felt that inflation will continue to be an important factor in financial calculations for the foreseeable future, and that any methodology used must have the capability of accurately accounting for its effects. It was felt that the preferred method of achieving this would be to perform (year-by-year) calculations in current (i.e., inflated) dollars. The alternative method of making computations in constant (uninflated) dollars was felt to require too many counter-Intuitive actions to be easily applicable. For instance, when working in constant dollars, Inflation cannot simply be ignored, because the depreciation schedule is fixed in current dollars and

* See Attachments. 
therefore must be deflated, or reduced in value by the amount of inflation.

\subsection{Choice of Discount Rate}

The discount rate to be used in the levelizing calculations is one of the most important parameters in the financlal model. It was generally recognized that the discount rate should be calculated on the basis of the financial structure and costs of the organization under consideration. This calculation, however, may be performed with or without considering the income tax deduction avallable from interest payments. The after-tax discount rate may be expressed as:

$$
R_{1}=(1-t) d r_{d}+(1-d) \tau_{e}
$$

where $t$ = effective income tax rate

$$
d=\text { proportion of debt (hence } 1-d=\text { proportion of equity) }
$$

$r_{\mathrm{d}}=$ Interest rate on debt

$\tau_{e}=$ required rate of return to equity.

The without-tax discount rate is given by:

$$
R_{2}=d r_{d}+(1-d) \tau_{e}
$$

It can be seen that $R_{1}$ will always be smaller than $R_{2}$, if $d$ is nonzero. Hence, assuming a cash-flow pattern which is initially heavily negative (due to e.g., investment in plant), the use of $R_{1}$ will lead to a lower levelized cost. (Numerical calculations indicate that the difference in costs could be larger than $15 \%$ for typical cases). 
There was no clear consensus among the workshop participants as

to whether $R_{1}$ or $R_{2}$ should be used. It appears that a majority of utility companies use $R_{2}$; the position in other industries is unknown.

The use of $R_{1}$ is valid only if the organization has sufficient income to take advantage of the interest tax deduction. However, under those circumstances it represents the true cost of capital better than $R_{2}$.

\subsection{Depreciation Method}

The clear feeling of the workshop participants was that the fastest possible depreciation schedule should be adopted for tax purposes. This will generally either be double declining balance, changing to stralght line half way through the project tax depreciation life, or sum-of-the-year's-digits.

Equipment lifetimes for tax depreciation purposes are not clear, since IRS has no rulings or guidelines for geothermal energy. Currently, IRS w111 allow 28 years $\pm 25 \%$ for steam plant depreclation life.

\subsection{Other Financial Assumptions and Parameters}

Parameters of prime concern here include debt/equity ratio, rate of return to equity, and interest rate on debt. Other elements of interest are tax rates (for both income and property taxes), inflation rates, and treatment of tax credits (e.g., depletion allowance, investment tax credit, etc.).

Two distinct views were expressed by the workshop participants 
concerning the proper debt/equity ratio for the field developer. Large energy resource companies, it was felt, will operate with debt/ equity ratios of about $25 / 75$ or lower. On the other hand, smaller developers, especially if covered by the loan guaranty program, may wel1 have debt/equity ratios near $75 / 25$, since the conditions of the loan guaranty program allow the proportion of equity capital to be as low as $25 \%$.

No reconciliation of these vlews was possible. It was felt that the cholce of debt/equity ratio for the developer should depend on the particular circumstances under consideration.

There was general agreement that debt/equity ratios of roughly 50/50 are appropriate for utilities. It must be noted that the equity portion is often divided into common and preferred equity, and the different rates of return for these types of stock must be taken account of when calculating the discount rate. Weighted (pre-tax) costs of capital of $15 \%$ to $20 \%$ were suggested for large energy resource companies, and $10 \%$ to $12 \%$ for utilities. These rates may change in the future as the result of changes in general financial conditions. The treatment of tax credits may depend upon the size of the organization being considered. Small companies with limited incomes may be forced to defer tax credits acquired during the development phase untll after plant start-up, when income will be available, while large companies can use credits against income from other ongoing projects. Because of discounting and inflation, deferred credits 
are less valuable than ones taken immediately, so that smaller companies will tend to have higher costs.

Conversely, smaller companies engaged in ofl and gas production are at present eligible for higher depletion allowances than larger companies.

Due to pending legislation, the exact tax credits and deductions which would be applicable to geothermal energy after 1978 were unknown at the time the workshop was held.

No consensus emerged from the workshop as to preferred assumptions and methods for handling tax credits. It was felt that the choice should be dependant upon the particular case under consideration. However, it was felt that assumptions used in analyses should be clearly stated in published material.

\subsection{Engineering Aspects}

It was recognized by the participants that the methodological questions involved in plant design and cost estimation are as complex and as important as those involved in financial analysis. It was generaily felt that a subsequent workshop could profitably be held to discuss engineering matters, such as field productivity and life, equipment lives, thermodynamic design methods, component cost est1mating, and $O \& M$ costs.

No detalled discussions took place in any of the above areas, except for well lives, and well replacement schedules.

Insofar as any consensus emerged, the feeling seemed to be that 
well lives would be at least 10 years, and that replacements would be necessary according to some relatively unpredictable decay/failure curve for wells. It was felt that a uniform rate of well replacement was too pessimistic in that probably fewer replacements would be needed in the early years of operation, but that Iumped replacement with all wells failing more-or-less together at the end of their life would be too optimistic, because wells could decline in productivity earlier. 


\subsection{CONCLUSIONS AND RECOMMENDATIONS}

The following conclusions emerged from the workshop sessions:

(1) The Division of Geothermal Energy at a minimum, and preferably the Department of Energy as a whole, should establish a standard financlal analysis methodology for use by all its contractors (the use of the standard methodology would be in addition to the use of whatever methodology the contractor chose for internal deciston making).

(2) This methodology should take the basic form of a year-by-year calculation method, with costs being expressed in current dollars. Either discounted cash flow or revenue requirements methodology would be generally acceptable.

(3) Where possible, guidelines should be established by DGE or DOE regarding the choice of financial parameters such as discount rate debt/equity ratio, treatment of taxes and tax credits, and equipment lifetimes, since widespread differences exist regarding preferred choices for these highly significant parameters.

(4) A similar workshop should be held to consider engineering aspects of geothermal electric generation, with particular emphasis being placed on thermodynamic design methods and cost estimating methods. 
ATTACHMENT A

LIST OF ATTENDEES 
LIST OF ATTENDEES

LEVELIZED BUSBAR COSTING METHODS WORKSHOP

The MITRE Corporation

June 29-30, 1978

NAME

KEITH MCKINNON

JAMES H. MALINOWSKI

EVERSON W. HULL

THOMAS A. V. CASSEL

MICHAEL R. ERVOLINI

LINDA FASSBENDER

C. R. RAO

TOD LARSON

YUE CHAU WONG

\section{ADDRESS}

SDG\&E

P.O. Box 1831

San Diego, California 92112

PG\&E

77 Beale Street

San Francisco, California 94611

TRW, INC.

7600 Colshire Drive

Westgate Park

McLean, Virginia 22102

University of Pennsylvania

Energy Center

3221 Walnut Street

Philadelphia, Pennsylvania 19104

University of Pennsylvania

Energy Center

3221 Walnut Street

Philadelphia, Pennsylvania 19104

Battelle Northwest

P.0. Box 999

Richland, Washington 19104

New Mexico State UnIversity

Box 3EI

Las Cruces, New Mexico 88003

Science Applications, Inc.

1200 Prospect

La Jolla, California

Bechtel National, Inc.

Box 3965

San Francisco, California 94119 
$\therefore \quad$ NAME

JOHN G. LEIGH

INJA K. PAIK

HARPAL S. DHILLON

JAMES V. COTTLE

WILLIAM L. POPE
ADDRESS

The MITRE Corporation 1820 Dolley Madison Boulevard Mail Station W389

McLean, Virginia 22102

Division of Geothermal Energy Economic Analysis Branch Department of Energy 20 Massachusetts Avenue, NW Ma11 Station 3122C

Washington, DC 20545

The MITRE Corporation 1820 Dolley Madison Boulevard Mail Station W389

McLean, Virginia 22102

Comptrollers Analytical Division Standard 011 Company of California 225 Bush Street

San Francisco, California 94104

Lawrence Berkeley Laboratory Building 20, Room 2082

End of Hearst Avenue

Berkeley, California 94720 
ATTACHMENT B

PAPERS PRESENTED

AT THE WORKSHOP

(Some items retyped for clarity) 


\title{
BECHTEL NATIONAL INC. \\ RESEARCH \& ENGINEERING
}

\author{
IIFE CYCIE COSTING \\ U S I $\mathbf{N}$ THE \\ RES O UR C E P R O G A M
}

JUNE 1978 
BECHTEL NATIONAL INC.

RESEARCH \& ENGINEERING

\section{INTRODUCTION}

Bechtel National Inc. has been involved in the engineering and economic evaluations of many geothermal facilities. The capital cost and operating costs estimated are combined to yield the busbar cost of electric energy, using an interactive computer system for calculations. The Cost Engineering group has recently developed a life cycle costing computer program RESOURCE (Revenue from Energy Sales of Utility to Recover Capital and Expenses) to achieve this.

Iife cycle costing methods usually differ from utility to utility, as there are many ways to analyze the cash flows. In order to deal with the various utility models, many computational options have been built into the RESOURCE program. At the discretion of the program user, calculation is made according to one of the wide variety of common life cycle costing models.

The following description outlines the basic features of RESOURCE, its capabilities and its output.

\section{PROGRAM OUTPUT}

RESOURCE calculates the levelized production cost of energy and expresses the results both in thousands of dollars per year and mills per kilowatt-hour. The levelized energy cost is the amount of revenue which over the plant life would exactly compensate for the recovery of all invested capital, the required return on investment and the payment of all taxes and expenses. 
The types of capital involved in capital recovery and return on investment are:

- bonded debt

- preferred equity

- common equity

The various types of taxes and insurance included are:

- federal income tax

- state income tax

- ad valorem property tax

- revenue tax or royalty

- property insurance

The various types of expenses included are:

- operation and maintenance

- administrative and general

- fuel

o other expenses 
The program output consists of three yearly cash flow tables and a summary. Cash flow Table 1 is the Capital Balance Sheet which shows the yearly capttal return and recovery. The following is an example of the Table 1 output.

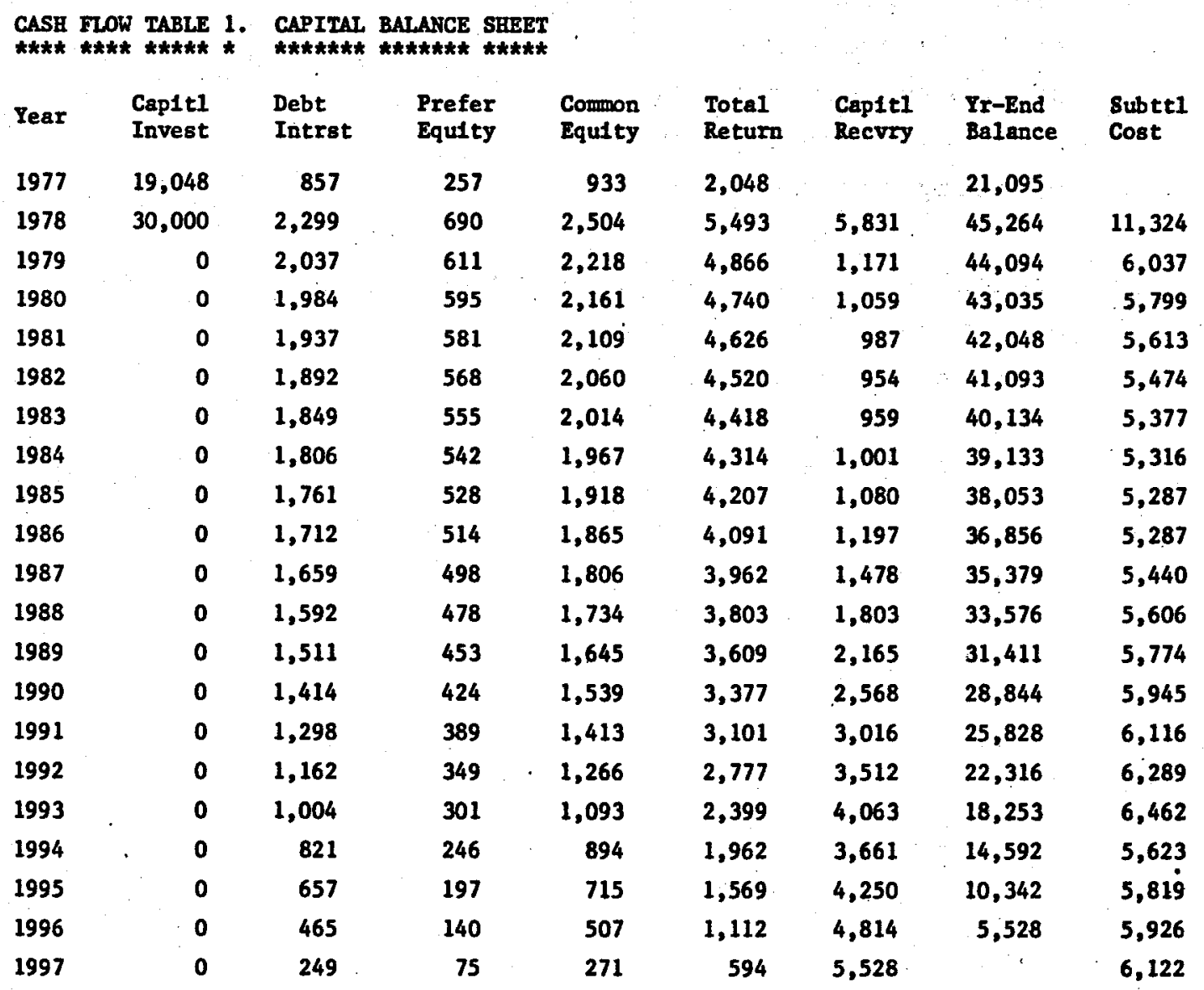


Cash flow Table 2, Taxes and Insurance, shows in 1ts varlous colums the income taxes, local taxes and Insurance. A sample printout of Table 2 1s shown below.

CASH FLOW TABIE 2. TAXES AND INSURANCE

$* * * * * * * * * * * * * * * * * * * * * * * * * * * * * * *$

\begin{tabular}{|c|c|c|c|c|c|c|c|c|c|}
\hline Year & $\begin{array}{l}\text { ED-TAX } \\
\text { DEPREC }\end{array}$ & $\begin{array}{l}\text { ST-TAX } \\
\text { DEPREC }\end{array}$ & $\begin{array}{l}\text { INV-IX } \\
\text { CREDIT }\end{array}$ & $\begin{array}{c}\text { FED } \\
\mathbf{I}-\mathbf{T A X}\end{array}$ & $\begin{array}{l}\text { STATE } \\
\text { IN-TAX }\end{array}$ & $\begin{array}{l}\text { REVENU } \\
\text { TAX }\end{array}$ & $\begin{array}{l}\text { PROPTY } \\
\text { TAX }\end{array}$ & $\begin{array}{l}\text { INSURE } \\
\text { PRMIUM }\end{array}$ & $\begin{array}{l}\text { SUBTIL } \\
\text { cosT }\end{array}$ \\
\hline 1978 & 6,131 & 5,450 & 4,905 & $-7,553$ & -478 & 78 & 1,022 & 182 & $-6,829$ \\
\hline 1979 & 5,365 & 4,844 & 0 & $-1,260$ & - 208 & 82 & 1,022 & 97 & $-\quad 267$ \\
\hline 1980. & 4,694 & 4,306 & 0 & -812 & -129 & 86 & 1,022 & 92 & 259 \\
\hline 1981. & 4,107 & 3,828 & 0 & $-\quad 397$ & 54 & 90 & 1,022 & 87 & 747 \\
\hline 1982 & 3,594 & 3,402 & 0 & -11 & 17 & 94 & 1,022 & 82 & 1,204 \\
\hline 1983 & 3,145 & 3,024 & 0 & 353 & 85 & 99 & 1,022 & 77 & 1,636 \\
\hline 1984 & 2,752 & 2,688 & 0 & 700 & 150 & 104 & 1,022 & 72 & 2,048 \\
\hline 1985 & 2,408 & 2,390 & 0 & 1,032 & 214 & 109 & 1,022 & 66 & 2,445 \\
\hline 1986 & 2,107 & 2,124 & 0 & 1,355 & 278 & 115 & 1,022 & 61 & 2,831 \\
\hline 1987 & 2,107 & 1,888 & 0 & 1,546 & 340 & 121 & 1,022 & 56 & 3,084 \\
\hline 1988 & 2,107 & 1,888 & 0 & 1,760 & 384 & 127 & 1,022 & 51 & 3,344 \\
\hline 1989 & 2,107 & 1,888 & 0 & 1,991 & 432 & 133 & 1,022 & 46 & 3,623 \\
\hline 1990 & 2,107 & 1,888 & 0 & 2,238 & 483 & 140 & 1,022 & 41 & 3,923 \\
\hline 1991. & 2,107 & 1,888 & 0 & 2,503 & 537 & 147 & 1,022 & 36 & 4,245 \\
\hline 1992 & 2,107 & 1,888 & 0 & 2,788 & 596 & 154 & 1,022 & 31 & 4,590 \\
\hline 1993 & 2,107 & 1,888 & 0 & 3,093 & 659 & 162 & 1,022 & 26 & 4,961 \\
\hline 1994 & 0 & 1,888 & 0 & 4,432 & 727 & 170 & 1,022 & 20 & 6,371 \\
\hline 1995 & 0 & 1,888 & 0 & 4,765 & 795 & 178 & 1,022 & 15 & 6,775 \\
\hline 1996 & 0 & D & 0 & 5,040 & 1,038 & 187 & 1,022 & 10 & 7,298 \\
\hline 1997 & 0 & 0 & 0 & 5,422 & 1,117 & 196 & 1,022 & 5 & 7,762 \\
\hline
\end{tabular}


Cash flow Table 3, Expenses and Revenue, shows the various operating expenses, total revenue requirements and present worths. The following is a sample Table 3 printout.

\begin{tabular}{|c|c|c|c|c|c|c|c|c|c|}
\hline $\begin{array}{l}\text { CASH } I \\
* * * *\end{array}$ & 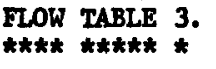 & $\begin{array}{l}\text { EXPEINSES } \\
\star * * \star \star \star \star \star *\end{array}$ & $\begin{array}{l}\text { AND REV } \\
* * * * * *\end{array}$ & & & & & & \\
\hline YEAR & $\begin{array}{l}\text { OPERAT } \\
\text { \& MAIN }\end{array}$ & $\begin{array}{l}\text { ADMINN } \\
\& \text { GEN }\end{array}$ & $\begin{array}{l}\text { FUEL } \\
\text { COST }\end{array}$ & $\begin{array}{l}\text { OTHER } \\
\text { EXPENS }\end{array}$ & $\begin{array}{c}\text { SUBTTL } \\
\text { COST }\end{array}$ & $\begin{array}{r}\text { TOTAL } \\
\text { REVENU }\end{array}$ & $\begin{array}{c}\text { P-W } \\
\text { FACTOR }\end{array}$ & $\begin{array}{c}\text { REVENU } \\
\text { P-W }\end{array}$ & $\underset{P \rightarrow W}{\text { CUMALL }}$ \\
\hline 1978 & 1,022 & 255 & 1,000 & 1,000 & 3,277 & 7,772 & .92268 & 7,171 & 7,171 \\
\hline 1979 & 1,073 & 268 & 1,050 & 0 & 2,391 & 8,161 & .85135 & 6,947 & 14,119 \\
\hline 1980 & 1,127 & 282 & 1,103 & 0 & 2,511 & 8,569 & .78552 & 6,731 & 20,849 \\
\hline 1981 & 1,183 & 296 & 1,158 & 0 & 2,636 & 8,997 & .72479 & 6,521 & 27,370 \\
\hline 1982 & 1,242 & 311 & 1,216 & 0 & 2,768 & 9,447 & .66875 & 6,318 & 33,688 \\
\hline 1983 & 1,304 & 326 & 1,276 & 0 & 2,907 & 9,919 & .61705 & 6,121 & 39,809 \\
\hline 1984 & 1,369 & 342 & 1,340 & 0 & 3,052 & 10,415 & .56934 & 5,930 & 45,738 \\
\hline 1985 & 1,438 & 359 & 1,407 & 0 & 3,205 & 10,936 & .52532 & 5,745 & 51,483 \\
\hline 1986 & 1,510 & 377 & 1,477 & 0 & 3,365 & 11,483 & .48471 & 5,566 & 57,049 \\
\hline 1987 & 1,585 & 396 & 1,551 & 0 & 3,533 & 12,057 & .44723 & 5,392 & 62,441 \\
\hline 1988 & 1,665 & 416 & 1,629 & 0 & 3,710 & 12,660 & .41265 & 5,224 & 67,665 \\
\hline 1989 & 1,748 & 437 & 1,710 & 0 & 3,895 & 13,293 & .38075 & 5,061 & 72,727 \\
\hline 1990 & 1,835 & 459 & 1,796 & 0 & 4,090 & 13,957 & .35131 & 4,903 & 77,630 \\
\hline 1991 & 1,927 & 482 & 1,886 & 0 & 4,294 & 14,655 & 32415 & 4,750 & 82,380 \\
\hline 1992 & 2,023 & 506 & 1,980 & 0 & 4,509 & 15,388 & .29909 & 4,602 & 86,983 \\
\hline 1993 & 2,124 & 531 & 2,079 & $\mathbf{0}$ & 4,735 & 16,157 & .27596 & 4,459 & 91,442 \\
\hline 1994 & 2,231 & 558 & 2,183 & 0 & 4,971 & 16,965 & .25463 & 4,320 & 95,762 \\
\hline 1995 & 2,342 & 586 & 2,292 & o & 5,220 & 17,814 & .23494 & 4,185 & 99,947 \\
\hline 1996 & 2,459 & 615 & 2,407 & 0 & 5,481 & 18,704 & .21678 & 4,055 & 104,001 \\
\hline 1997 & 2,582 & 646 & 2,527 & o & 5,755 & 19,639 & .20002 & 3,928 & 107,929 \\
\hline
\end{tabular}


The final table, Summary, shows the levelized costs of Individual Items as well as the total levelized cost of electric energy. When escalation of energy cost is taken into account in the calculations, the levelized energy cost will be expressed in terms of the initial cost.

SUMMARY TABLE OF LEVELIZED COSTS

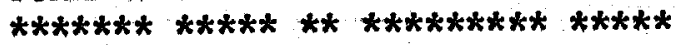
COST ITEM

Return on Investment

$\begin{array}{rc}\frac{\text { SK/YR }}{2,759} & \frac{(M I L / R W H)}{8.39} \\ 1,529 & 4.65 \\ 314 & .96 \\ 78 & .24 \\ 703 & 2.14 \\ 46 & .14 \\ 1,022 & 3.11 \\ 255 & .78 \\ 1,000 & 3.04 \\ 66 & .20 \\ -7,772 & 23.65^{*}\end{array}$

TOTAL ENERGY PRODUCTION COST

Capital Recovery

Income Taxes

Gross Revenue Tax

Property Tax

Property Insurance

Operation \& Maintenance

Administration \& General

Fue1

Other Expenses General 
PROGRAM INPUT

RESOURCE is a flexible program which provides various options for treating capital recovery, discount rate, tax depreciation, AFDC, etc. The user selects a specific methodology by his interactive input to the computer. The options established are described below.

\section{Capital Recovery Options}

- Use the sinking fund method for book depreciation and calculate the uniform revenue requirement.

- Use the straight-line method for book depreciation and calculate revenue requirements year by year.

\section{Discount Rate Options}

- Use the after-tax discount rate, ie., the rate of return on investment less the tax credit for interest.

- Use the rate of return on investment as the discount rate.

- Use a discount rate specified by the user.

\section{Income Taxes Options}

- Allow negative income taxes in the calculations.

- Allow tax losses and unused investment tax credit to be carried over to subsequent years in accordance with IRS guidelines.

\section{Tax Depreciation Options}

- Use sum-of-the-year-digits method

- Use double-declining-balance method

- Use straight-line method

- Use different methods for different capital investmets, to be specified by the user. 
Property Tax Options

- Calculate property tax as a constant percentage of the original invested capital.

- Calculate property tax as a percentage of the capital depreciated linearly over the life of the plant and escalate according to the capital cost escalation rate.

\section{Operating Expenses Options}

- Calculate operation and maintenance costs as a percentage of the capital cost and administrative and general costs as a percentage of the operation and maintenance costs.

- Use operation and maintenance oosts and administrative and general costs over the life of the plant, specifically furnished by the user.

- Calculate operation and maintenance costs based on the installation schedules of production wells, injection wells and surface facilities specified by the user, and administrative and general costs as a percentage of the operation and maintenance costs. (This option is provided solely for geothermal wellfields).

\section{Escalation Options}

- Exclude escalation in the calculations.

- Use escalation rates for the capital cost, operation and maintenance, administrative and general, fuel and electric energy costs as specified by the user.

\section{AFDC Options}

- Calculate the return on investment during pre-operational years according to the established return rate over the plant life with debt interests deducted for income tax purposes in the first year of operation. 
- Use the effective AFDC rate during pre-operational years supplied by the user without specifying debt interest and equity return. (There is no income tax deduction since tax credits for the interest are taken into consideration in establishing the effective AFDC rate).

- Use the treatment of AFDC as above, but with the amount of AFDC included in the capital cost bases for tax depreciations during operational years. (Although this procedure is incorrect, it is used in some life cycle models, and is included as an option for comparative purposes).

\section{Financial and Tax Data Options}

- Use the set of data on finances, return rates and tax rates built in the program.

- Use data on finances, return rates and tax rates supplied by the user.

The above options provide an almost unlimited number of combinations Thus, RESOURCE is capable of performing life cycle costing calculations according to the economic models used by most organizations.

Once the life cycle costing model is chosen, the program user inputs numerical data to the computer on the interactive terminal. In the case that a set of data is. derived by the computer, as provided by some of the options, the numerical values are printed out on the terminal. 
Because of its flexibility and responsiveness to the users needs, RESOURCE is also ideally suited to sensitivity analysis. This is possible through a special feature built into the program which permits the user to quickly vary certain data points.

In summary, RESOURCE has been designed to perform economic evaluations so necessary to the emerging energy technologies.

R\&E Cost Engineering

Bechtel National Inc. 


\section{Bechtel Electric Energy Cost Estimating Programs}

- "Buscost" : Computes Levelized Energy Costs for Geothermal Well Field and Power Plant Using Electric Utility Methodology.

- "RESOURCE" : Computes Economic Parameters for Capital Plants Using Flexible Financial Methodologies. 
BECHTEL "BUSCOST" WELL FIELD ENERGY COST INPUT

Enter Life of Wells (Yrs): 10

Enter Life of Well-Field Surface Facilities (Yrs): 30

Enter Life of Power Plant (Yrs): 30

No. of Yrs in Which Investments Are Made: 3

Yr. Wel1 Investment (\$R), Surf.Fac.Investment (\$R): $1,4000,8000$

Yr. We1l Investment ( $\$ R)$, Surf.Fac.Investment (\$R): $11,4000,0$

Yr. Well Investment (\$R), Surf.Fac.Investment (\$R): $21,4000,0$

Yr: 1

Prod Wells Added, Reinj Wells Added: 8,4

Yr: 11

Prod Wells Added, Reinj Wells Added: 8,4

Yr: 21

Prod Wells Added, Reinj Wells Added: 8,4

Productive Exploration Costs ( $(R): 2000$

Cost of Capital Data

Percent Debt Financing, Annual Cost of Debt (\%): 0,0

Percent Equity Financing, Annual Cost of Equity (\%): 100,20

Weighted Avg Cost of Cap1tal: 20\%

Énter Present Value Discount Rate (\%): 20

Income Tax Data

Federal Tax Rate (\%): 48

State Tax Rate (\%): 9

Effective Tax Rate: $52.68 \%$

Investment Tax Credit (\%): 10

Royalties: \% Of Gross Revenues: 10

Annual O\&M Expense ( $\$ R$ ) - Enter 0 if to be Computed: 0

Admin \& Genl Expense as \% of O\&M: 10

Ad Valorem (Production) Tax as \% of Gross Revenues: 10 


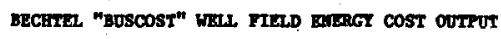

Exploration Costo Capitelized Yrow Year 1 (SK): 2000

\begin{tabular}{|c|c|c|c|c|c|c|c|c|c|c|c|c|c|c|c|}
\hline Ix & PR-KLS & $x w-n \Omega s$ & WL-INV & SP-ENV & BX-VAI & SR-DPR & ROI & 2X-DPR & INTRST & Ire & $\mathbf{n}-\boldsymbol{\tau} \mathbf{A x}$ & ooss & $A \in G$ & BortT & $A D-V$ \\
\hline 1 & 8 & 4 & 4000 & 8000 & 14000 & 732 & 2808 & 1466 & 0 & 746 & 721 & 1048 & 104 & 673 & 675 \\
\hline 2 & 0 & 0 & 0 & 0 & 13268 & 732 & 2653 & 1261 & 0 & 653 & 984 & 1048 & 104 & 690 & 690 \\
\hline 3 & 0 & 0 & 0 & o & 12536 & 732 & 2507 & 2092 & 0 & 0 & 2390 & 1048 & 104 & 847 & 847 \\
\hline 4 & 0 & 0 & 0 & 0 & 11804 & 732 & 2360 & 950 & 0 & 0 . & 2384 & 1048 & 104 & 828 & 828 \\
\hline 5 & 0 & 0 & 0 & 0 & 11072 & 732 & 2214 & 832 . & 0 & 0 & 2353 & 1048 & 104 & 806 & 806 \\
\hline 6 & 0 & 0 & 0 & 0 & 10360 & 732 & 2068 & 733 & 0 & 0 & 2301 & 1048 & 104 & 781 & 781 \\
\hline 7 & 0 & 0 & 0 & 0 & 9608 & 732 & 1921 & 702 & 0 & 0 . & 2171 & 1048 & 106 & 747 & 747 \\
\hline 8 & o & 0 & 0 & 0 & 8876 & 732 & 1775 & 673 & 0 & 0 & 2041 & 1048 & 104 & 712 & 712 \\
\hline 9 & 0 & 0 & 0 & 0 & 8144 & 732 & 1628 & 645 & 0 & 0 & 1909 & 1048 & 104 & 677 & 677 \\
\hline 20 & 0 & 0 & 0 & 0 & 7412 & 732 & 1482 & 619 & 0 & 0 & 1775 & 1048 & 104 & 642 & 642 \\
\hline 21 & 8 & 4 & 4000 & 0 & 20680 & 732 & 2136 & 1133 & 0 & 400 & 1086 & 1048 & 104 & 638 & 638 \\
\hline 12 & 0 & 0 & 0 & 0 & 9948 & 732 & 1989 & 951 & 0 & 0 & 1970 & 1048 & 104 & 730 & 730 \\
\hline 13 & 0 & 0 & 0 & 0 & 9216 & 732 & 1843 & 803 & 0 & 0 & 1972 & 1048 & 104 & 712 & 712 \\
\hline 14 & 0 & 0 & 0 & o & 8484 & 732 & 1696 & 680 & 0 & 0 & 1945 & 1048 & 204 & 690 & 690 \\
\hline 15 & 0 & 0 & 0 & 0 & 7752 & 732 & 1530 & 580 & 0 & 0 & 1894 & 1048 & 104 & 666 & 666 \\
\hline 16 & 0 & 0 & 0 & 0 & 7020 & 732 & 1404 & 498 & 0 & 0 & 1823 & 1048 & 104 & 638 & 638 \\
\hline 17 & 0 & 0 & 0 & 0 & 6288 & 732 & 1257 & 498 & 0 & 0 & 2659 & 1048 & 104 & 600 & 600 \\
\hline 18 & 0 & 0 & 0 & 0 & 5536 & 732 & 1111 & 498 & 0 & 0 & 1697 & 1048 & 104 & 561 & 561 \\
\hline 19 & 0 & 0 & 0 & 0 & 4826 & 732 & 964 & 498 & 0 & 0 & 1333 & 2068 & 104 & 522 & 522 \\
\hline 20 & 0 & 0 & 0 & 0 & 4092 & 732 & 818 & 498 & 0 & 0 & 1111 & 1048 & 104 & 484 & 484 \\
\hline 21 & 8 & 4 & 4000 & 0 & 7360 & 732 & 1472 & 1036 & 0 & 434. & $454^{\circ}$ & 1040 & 104 & 476 & 476 \\
\hline 22 & 0 & 0 & 0 & o & 6628 & 732 & 1325 & 876 & o & 0 & 1314 & 1048 & 104 & 365 & 565 \\
\hline 23 & 0 & 0 & 0 & 0 & 5696 & 732 & 2179 & 740 & 0 & 0 & 1294 & 1048 & 104 & 544 & 544 \\
\hline 24 & 0 & 0 & 0 & 0 & 5164 & 732 & 1032 & 645 & 0 & 0 & 2245 & 1048 & 204 & 520 & 520 \\
\hline 25 & 0 & 0 & 0 & 0 & 4432 & 732 & 886 & 563 & 0 & 0 & i114 & 1048 & 204 & 493 & 493 \\
\hline 26 & 0 & 0 & 0 & 0 & 3700 & 732 & 740 & 498 & 0 & 0 & 1084 & 1048 & 104 & 463 & 463 \\
\hline $\begin{array}{l}27 \\
28\end{array}$ & ${ }_{0}^{n}$ & 0 & 0 & 0 & 2968 & 732 & 593 & 498 & 0 & 0 & 920 & 1048 & 104 & 424 & $\begin{array}{l}.124 \\
.286\end{array}$ \\
\hline .29 & 0 & 0 & $\begin{array}{l}0 \\
0\end{array}$ & $\begin{array}{l}0 \\
0\end{array}$ & $\begin{array}{l}2236 \\
1504\end{array}$ & $\begin{array}{l}732 \\
732\end{array}$ & $\begin{array}{l}447 \\
300\end{array}$ & $\begin{array}{l}498 \\
498\end{array}$ & $\begin{array}{l}0 \\
0\end{array}$ & $\begin{array}{l}0 \\
0\end{array}$ & $\begin{array}{l}758 \\
594\end{array}$ & $\begin{array}{l}1048 \\
1048\end{array}$ & $\begin{array}{l}104 \\
104\end{array}$ & $\begin{array}{l}386 \\
347\end{array}$ & 347 \\
\hline 30 & 0 & 0 & 0 & 0 & 772 & 732 & 154 & 490 & 0 & 0 & 431 & 1048 & 104 & 308 & 308 \\
\hline
\end{tabular}

*** LEVEL AmRon costs (SR) ***

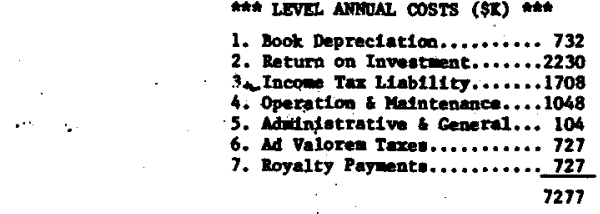


BECHTEL "BUSCOST" POWER PLANT ENERGY COST INPUT

Enter Expected Plant Life (Yrs): 30

Enter Beginning-of-Yr Investment Schedule

No. of Investments During Plant Life: 1

Yr of Plant Life, Capital Invested (\$K): 1,50000

Cost of Capital Data

Percent Debt Financing, Annual Cost of Debt (\%): 50, 9.5

Percent Equity Financing, Annual Cost of Equity (\%): 50, 12.5

Weighted Avg Cost of Capitai: 11\%

Enter Present Value Discount Rate (\%): 11

Income Tax Data

Note: Following 3 Tax Rate Inputs are 0 for Publicly-Owned Utility

Federal Tax Rate (\%): 48

State Tax Rate (\%): 9

Effective Tax Rate: $52.68 \%$

Investment Tax Credit (\%): 10

O\&M as $\%$ of Capital cost $=2 \%$ Typica1 $(\%): 2$

Real, Uninflated Annual. O\&M Escalation Rate (\%): 0

Admin \& Ganl As \% of O\&M = 25\% Typical (\%): 25

Ad Valorem Tax as \% of Capital cost $=2.5 \%$ Typical (\%): 2.5

Plant Insurance as $\%$ of Capital Cost $=0.1 \%$ Typical $(\%): .1$ 
BECHTEL "BUsCosT" POWER PLART ENERGT COST OUTPUT

\begin{tabular}{|c|c|c|c|c|c|c|c|c|c|c|}
\hline $\begin{array}{l}3 x-V A L \\
50000\end{array}$ & $\begin{array}{c}3 x-0 P R \\
1666\end{array}$ & $\begin{array}{l}801 \\
5500\end{array}$ & $\begin{array}{c}\text { IX-DPR } \\
3333\end{array}$ & $\begin{array}{c}\text { INTRST } \\
2375\end{array}$ & $\begin{array}{l}\text { ITC } \\
529\end{array}$ & $\begin{array}{c}\text { IS-TAX } \\
\text { 504: }\end{array}$ & $\begin{array}{l}0601 \\
1000\end{array}$ & $\begin{array}{l}\Delta 5 G \\
250\end{array}$ & $\begin{array}{c}\text { AD-TAX } \\
1250\end{array}$ & $\begin{array}{r}\text { INS } \\
50\end{array}$ \\
\hline 48334 & 1666 & 5316 & 3111 & 2295 & 571 & 546 & 1000 & 250 & 1250 & 50 \\
\hline 46668 & 1666 & 5133 & 2903 & 2216 & 608 & 583 & 1000 & 250 & 1250 & 50 \\
\hline 45002 & 1666 & 4950 & 2710 & 2137 & 640 & 625 & 1000 & 250 & 1250 & 50 \\
\hline 43336 & 1666 & 4766 & 2529 & 2058 & 667 & 642 & 1000 & 250 & 1250 & 50 \\
\hline 41670 & 1666 & 4583 & 2360 & 1979 & 691 & 666 & 1000 & 250 & 1250 & so \\
\hline 40004. & 1666 & 4400 & 2203 & 1908 & 709 & 684 & 1000 & 250 & 1250 & 50 \\
\hline 38338 & 1666 & 4217 & 2056 & 1821 & 580 & 1005 & 1000 & 250 & 1250 & 50 \\
\hline 36672 & 1666 & 4033 & 1919 & 1741 & 0 & 2269 & 1000 & 250 & 1250 & 50 \\
\hline 35006 & 1666 & 3850 & 1791 & 1662 & 0 & 2296 & 1000 & 250 & 1250 & 50 \\
\hline 33340 & 1667 & 3667 & 1671 & 1583 & 0 & 2315 & 1000 & 250 & 1250 & 50 \\
\hline 31673 & 1667 & 3484 & 1560 & 1504 & 0 & 2323 & 1000 & 250 & 1250 & 50 \\
\hline 30006 & 1667 & 3300 & 1456 & 1425 & 0 & 2322 & 1000 & 250 & 1250 & 50 \\
\hline 28339 & 1667 & 3117 & 1359 & 1346 & 0 & 2314 & 1000 & 250 & 1250 & 50 \\
\hline 26672 & 1667. & 2933 & 1268 & 1266 & 0 & 2300 & 1000 & 250 & 1250 & 50 \\
\hline 25005 & 1667 & 2750 & 1183 & 1187 & 0 & 2278 & 1000 & 250 & 1250 & 50 \\
\hline 23338 & 1667 & 2567 & 1183 & 1108 & 0 & 2163 & 1000 & 230 & 1250 & 50 \\
\hline 21671 & 1667 & 2383 & 1183 & 1029 & 0 & 2046 & 1000 & 250 & 1250 & so \\
\hline 20004 & 1667 & 2200 & 1183 & 950 & 0 & 1930 & 1000 & 250 & 1250 & 50 \\
\hline 18337 & 1667 & 2017 & 1183 & 871 & 0 & 1814 & 1000 & 250 & 1230 & 50 \\
\hline 16670 & 1667 & 1833 & 1183 & 791 & 0 & 1698 & 1000 & 250 & 1250 & 50 \\
\hline 15003 & 1667 & 1650 & 1183 & 712 & 0 & 1583 & 1000 & 250 & 1250 & 50 \\
\hline 13336 & 1667 & 1466 & 1183 & 633 & 0 & 1456 & 1000 & 250 & 1250 & 50 \\
\hline 11669 & 1667 & 1283 & 1183 & 354 & 0 & 1350 & 1000 & 250 & 1250 & 50 \\
\hline 10002 & 1667 & 1100 & 1183 & 475 & 0 & 1236 & 1000 & 250 & 1250 & 50 \\
\hline 8335 & 1667 & 916 & 1183 & 395 & 0 & 1118 & 1000 & 250 & 1250 & 50 \\
\hline 6668 & 1667 & 733 & 1183 & 316 & 0 & 1003 & 1000 & 250 & 1250 & 50 \\
\hline 5001 & 1667 & 550 & 1183 & 237 & 0 & 887 & 1000 & 250 & 1250 & 50 \\
\hline 3334 & 1667 & 366 & 1183 & 158 & 0 & 770 & 1000 & 250 & 1250 & 50 \\
\hline 1667 & 1667 & 183 & 1183 & 79 & 0 & 654 & 1000 & 250 & 1250 & so \\
\hline
\end{tabular}

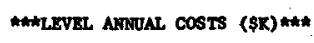

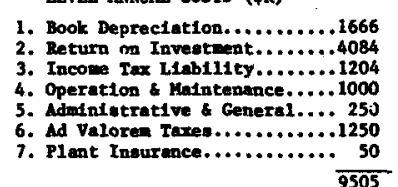




\section{BECHTEL "BUSCOST" WELL FIELD EXPLORATION COSTS}

Costs on Retained Acreage for 200 MWe Producing Area.

\section{ITEM}

Geological and Geophysical Studies

Land (estimate 7500 scres ( \$7)

1 Exploratory well

3 Step-out wells \& $\$ 365,000$

Testing to establish potential
1975 COSTS

$\$ 85,000$

53,000

410,000

$1,095,000$

540,000

$\$ 2,183,000^{\circ}$ 


\section{BeCHTEL "BUSCOST" WELL FIELD ANNUAL 0 \& M ExPENSES}

$\$ 70,000$

$\$ 250,000 \times\left(\frac{\text { No.Prod.Wel1s }}{13}\right)^{0.3}$

$\$ 50,000 \times$ (No.Prod.Wells)

$\$ 80,000 \times$ (No.Reinj.Wells)

$0.5 \% \times$ (Surface Piping Capital)
: Foremen \& Site Managers

: Manual \& Technical Labor \& Operating Materials

: Production Well Maintenance

: Reinjection Well Maintenance 


\section{BECHTEL "BUSCOST" InCOME TAX Equations}

- Combined State \& Federal Income Tax Liability in Year " $n$ ":

$T L_{n}=\frac{T\left(C R+R O I_{n}-D T_{n}-B_{n}\right)-I T C_{n}}{I-T}$

- Effective State \& Federal Tax Rate:

$$
T=T_{s}+T_{f}\left(1-T_{s}\right)
$$

- Tax Depreciation in Year " $n$ ":

$$
\left(c_{0}-\sum_{i=1}^{n-1} D T_{1}\right) \times(2 / L) \quad \text { for } \frac{2}{L}>\frac{1}{L-(n-1)}
$$

$D T_{n}=$

$$
\left(c_{0}-\sum_{i=1}^{n-1} D T_{1}\right) /(L-(n-1)) \quad \text { for } \frac{2}{L} \leq \frac{1}{I-(n-1)}
$$


BeCHTEL "Bu sCost" Energy COSt LeVelizing Technique

$$
\sum_{i=1}^{L} \bar{P} E_{i} P W F_{1}=\sum_{1=1}^{L}\left(\sum_{n=1}^{7} C_{n, 1}\right) \quad P W F_{1}
$$

$\stackrel{\omega}{\infty}$

$$
\bar{p}=\frac{\sum_{i=1}^{L}\left(\sum_{n=1}^{7} c_{n, 1}\right) P W F_{1}}{\sum_{1=1}^{L} E_{1} P W E_{1}}
$$

$$
\mathrm{PWF}_{i}=\frac{1}{(1+r)^{i}} \quad \mathrm{E}_{i}=\mathrm{P}_{i} \times \mathrm{CF} \times 8.760 \times 10^{6}
$$




\section{BECHTEL "BUSCOST" A.E.D.C. EstIMATES FOR GEOTHERMAL PLANTS}

SCHEDULE DURATION AND ALLOWANCE FOR FUNDS DURING CONSTRUCTION (AFDC) FOR GEOTHERMAL POWER PLANTS

\begin{tabular}{|c|c|c|}
\hline $\begin{array}{c}\text { Geothermal Power } \\
\text { plant Capacity }\end{array}$ & $\begin{array}{l}\text { Englneering and } \\
\text { Construction } \\
\text { Schedule Duration }\end{array}$ & $\begin{array}{l}\text { AFDC as Percent ( } 1) \\
\text { of Project Cost }\end{array}$ \\
\hline 10 MWe & 34 Months. & $9.4 \%$ \\
\hline $\begin{array}{c}50 \mathrm{MWe} \\
(1-50 \mathrm{MWe} \text { Untt) }\end{array}$ & 40 Months & $11.2 \%$ \\
\hline $\begin{array}{c}100 \mathrm{MWe} \\
(2-50 \mathrm{MWe} \text { Units })\end{array}$ & 48 Nonths & $13.6 \%$ \\
\hline $\begin{array}{c}200 \mathrm{MHe} \\
\text { (4-50 } \mathrm{MWe} \text { Units) }\end{array}$ & 58 Months & $16.6 \%$ \\
\hline
\end{tabular}

(1) Project cost includes engineering, construction and owner's ... costs. AFDC based on 10\% annual rate applied to project cost and compounded annually from center-of-gravity of cash flow schedule to beglnning of commercial operation 
Dr. Momtaz Mansour

\section{SUIIPARY}

GEOCOST combines both technical and economic factors into one systematic cost accounting framework. The program, which simulates production of electricity from most types of geothermal resources, is composed of trio parts: a model that simulates the costs associated with the exploration, development, and operation of a geothermal reseroviri and a model that simulates the costs associated with the design, construction, and operation of the power plant. The costs from the reservoir model become the energy supply costs to the power plant. The combined reservoir and power plant models form the complete energy production system. GEOCOST can simulate the financial and tax structure of almosit any corporate or economic entity through varying rates of return on equity and debt, the debt-equity ratios, and tax rates.

Generation of electricity from geothermal energy involves a sequence of costs and revenues. Starting with exploration for a geothermal reservoir, the process continues through the location and development of the reservoir, power plant construction, and power production, and ends when either the power piant or the reservoir reaches the end of its useful life.

These events can be represented by a sequence of cash flows which form the basis for the GEOCOST model. Using discounted cash flow analysis, the program calculates the costs of energy by equating the present worth of the revenues and expenses over the economic life of the plant or reservoir. $\therefore \cdots$

Welihead conditions of the geothermal fluid, power plant design and capacity, cost of the wells, and annual power production are the principal input requirements. The two main components of the program, the reservoir and the power plant models, are linked through the fluid transmission and the power plant system submodels. The latter calculates the total fluid flow requirements from the reservoir at the wellhead conditions. From this total flow, the fluid transmission. submodel calculates the number of wells required, pipe lengths and diameters, pumping requirements, and the temperature and pressure drop between the reservoir and the power plant. The program iterates between the power.plant systems submodel (which calculates the additional flow required under the degraded fluid conditions) and the fluid transmission submodel until the successive flow requirements converge within ${ }^{\circ}$ preset criteria. 
Dr. Momtaz Mansour

After establishing the total fluid flow that meets the specified power production requirements, the program determines the cash flow for the reservoir. This includes capital-costs and operating expenses from the beginning of exploration through the life of the power piant. Based on the cash flow and energy supplied, the unit cost of energy is calculated that will equate the present worth of the revenues and expenses for the reservoir. The usefut ilfe of the power plant is the operating period of the reservoir since energy must be supplied throughout this period.

In the power plant model the revenue to the reservoir is the energy supply expense to the power plant. This expense is combined with the power plant capital costs and operating expenses to generate the cash flow for the plant. Based on the power. plant cash flow and the electricity generated, the unit cost of electricity is calculated by equating the present worth of the revenues and expenses. 
GEOTHERMAL DEVELOPMENT AND THE PRODUCTION OF ELECTRICITY

Summary

\section{Introduction}

The purpose of the following presentation is to describe the logic underlying research performed, as well as the tools used and the results of that research concerning the potential contribution of geothermal development to electricity production in the five state area Including New Mexico, Arizona, Nevada, Utah, and Colorado. The following section outilnes the underlying philosophy of the research program. The next section discusses the simulation model used to evaluate the potential profitability of Geothermal sites for electric use, the geothermal Internal Rate of Return Algorithm (GIRORA-Electric). The third section following this introduction presents a discussion of the sensitivity of profits to changes in physical and policy variables circumscribing the development of geothermal electric power. The section following the discussion of profit sensitivity analysis presents site by site profitability as determined by use of GIRORA-Electric, and aggregation of resulting power estimate and a discussion of power-on-line profections. In addition, the sensitivity of power-onIne estimate to policy inftiatives is discussed. Finally, preliminary estimates of electric power supply and demand conditions are presented. The feed back or impact upon the development of geothermal electric plants is discussed under three posstble 
sets of alternative assumptions. We turn first to a discussion of the philosophy underlying the research accomplished concerning the development of geothermal electric power.

\section{Research Philosophy}

Underlying all considerations of this research was the assumption that electric plants driven by geothermal energy, and the flelds which deliver that energy, will be developed by the private or non-Federal government sectors. For the most part, this means private developers in conjunction with regulated electric utilities (although, exceptions may and will likely exist). A concomitant of this assumption is that a geothermal resource will be developed only if it is economic to do so (that is, economic feasibility is a necessary, but not sufficient condition). Because private developers and electric utilities are the actors, it is necessary to consider economic feasibility (profitability) from their point-of-view.

The second principal assumption is that geothermal energy is only one of a variety of energy sources at the disposal of an electric utility (or anyone, for that matter, in need of electric power). A concomitant of this second assumption is that geothermal energy must compete In the market place for energy. Because geothermal energy is a marginal energy source, marginal in the sense the it is a relatively small source, and in this region enters the scene chronologically late, it must compete at prices established by other fuels. (coal, nuclear, oil, gas, and hydroelectric).

The result of the first two principal assumptions is that if geothermal energy Is not profltable for both the geothermal developer and the electric utility, at a price which enables the electric power thus generated to be competitive with that generated by other fuels, the resource will not be developed. This is the bottom 1ine, Given other institutional, attitudinal, and economic factors, a particular resource may not be developed, even if it is economically competitive. 
Moreover, even though a particular resource is economically competitive and faces no noneconomic barriers, being a marginal fuel it may not be developed for lack of effective demand. This could occur under two conditions: (1) increases in the price of electricity result in sufficient consumer substitutions to reduce quantity of electricity demanded below some critical level, or (2) general economic growth is Insufficlent to require the capacity that would be added by a geothermal development. The first condition may have some general applicability, while the second might occur only in remote, sparsely populated areas (due to the portablilty of electricity).

At each juncture, government policy makers may have an option to affect the environment in which private developers make decisions or perceive opportunities. It was the aim of the research effort described below (1) to ascertain what elements under the influence of DOE-DGE could efficlently alter the profitability of geothermal resources as perceived by the investors, (2) to Identify the magnitude of Impact policy action might reasonably be expected to have, and (3) to estimate in a preliminary way the level of market potential for electricity driven by geothermal energy.

Because geothermal energy is not to be had for free, it is an economic resource which should be developed efficiently with due concern for its place in a total energy perspective. Private entrepreneurs cannot discount the value of national balance of payments deficits, or other social concerns when considering the desirability of an investment. It must be DOE-DGE's role to consider this broader perspective and insure the avallability of sufficlent incentives (or the absence. of disincentives) so that private entrepreneurs will percelve geothermal energy as an attractive opportunity.

The criterion for evaluation chosen for this research is the rate-of-return on equity investment. There are a variety criteria which could be used, each having ther own special strengths and weaknesses. The one chosen for this research 
seems most appropriate because of the overall capital intensity of the process. It should be added that in the process of ranking geothermal sites, or judging the relative effectiveness of policy actions, any criterion of profitability or economic feasibility would yield approximately the same answer as another, so long as the methodology is consistent.

As will be noted in the next section, the simulation model used to evaluate geothermal electric projects uses the rate-of-return as the basis for comparison rather than the price of electricity. This is due to our assumption that geothermal energy must compete at the margin with established fuels. The price of electriclty is then an independent rather than dependent variable. Moreover, using a rate-of-return on equity as the dependent variable focuses our attention on the profit motive of the developer. The developer can invest in geothermal energy, or any other income yielding prospect. If the rate-of-return on geothermal energy is not high enough (at a price where it can effectively compete), the developer will place Investments elsewhere.

\section{Summary of Geothermal Algorithm - Electric Uses}

The model which follows posits the very simple economic idea that an investment should be made if the present value of all net income streams is equal to the Investment required to generate them when discounted at an interest rate appropriately high. $(\mathrm{fn})$ If the amount of an investment is known, and the net income streams are known, the rate-of-return can be calculated. This model attempts to calculate the Internal rate-of-return to a producer from a geothermal field for which electricity production is planned. This model can easily be modified to ftt any type of energy production from a geothermal field.

The basic equation is of the simple form for discounting cash flows:

$$
\text { (1) Investment } \left.=\sum_{t=1}^{T} \text { (Net Income }\right)_{t}(1+R)^{-t}
$$

(fin)For a discussion of this principal, see Fred R. Glahe Macroeconomics (New York Harcourt, Brace, Jovanich, Inc., 1973), pp. 91-95. 
where Net Income $=$ (Total Revenues) - (Total Operating Cost) - (Taxes)

Total Revenues $=$ (Price) (Quantity Output)

Total Operating Cost $=$ A Percentage of Investment

R: Rate-of-Return

$t$ : Each year, $t=1,2, \ldots, T$.

T: Expected 1ife span of the Investment. However, this rather fundamental potion is modified somewhat in GIRORAElectric due to the fact that some investments are made throughout the life of the project and thus must also be discounted to the present for proper evaluation. This yields the equation,

$$
\text { (2) } \sum_{t=1}^{T}\left(\text { Investment } t_{t}\right)(1+R)^{-t}=\sum_{t=1}^{T}\left(\text { Net Income }{ }_{t}\right)(1 \pm R)^{-t}
$$

which reduces to

$$
\text { (3) } \left.\sum_{t=1}^{T} \text { (Investment }{ }_{t}-\text { Net } \text { Income }_{t}\right)(1+R)^{-t}=0
$$

If we set $(1+R)^{-1}=b$, then

(4) $\sum_{t=1}^{T}$ (Investment $t_{t}-$ Net Income $\left.{ }_{t}\right) b^{t}=0$

Equation (4) is a polynomial with $T$ roots. Assuming a monotonic function there w111 be one real (non-imaginary) root (b) which permits a solution for the equation. This root can be found iteratively by Newton's technique using a computerized program. ${ }^{(f n)}$ Fortunately, GIRORA-Electric has generated only monotonic functions. GIRORA-Electric simulates all investment, revenue, and cost flows for a specified resource site, and inputs the flows to equation (3) and solves for $R$.

(fn) For a description of the mathematical technique involved, see C. C. T, Baker, Dictionary of Mathematics, (New York City, Hart Publishing Co., 1966), p. 206. 
Investment, revenue and cost flows are divided into two sectors, a geothermal developer sector, and an electric utility sector. The geothermal developer's revenues are operating costs as perceived by the electric utility. The electric utility demand for geothermal fluid (or steam) is derived from the demand for electricity. Once a quantity of electricity has been specified, given resource characteristics, the quantity of fluld or steam is automatically specified.

The price of the geothermal energy is the highest price consistent with the competitive price of electricity and the electric utility's regulated rate-of return (both which are assumed to be known). The description of GIRORA-Electric begins with a description of the Electric Utillty Sector.

\section{Electric Utility Sector}

The model first Identifies the principal components of cost to the electric utility for each year $(t)$ of electricity production: capital costs $\left(\mathrm{CC}_{t}\right)$, operatIng costs $\left(\mathrm{OMC}_{t}\right)$, income taxes $\left(\operatorname{ITX}_{t}\right)$, property taxes $\left(\operatorname{PTX}_{t}\right)$, and energy costs $\left(\right.$ ENC $\left._{t}\right)$.

Capital costs are a function the investment $(K)$ required for a specified level of capacity in kilowatts (CAP).

(1) $\mathrm{K}=(\mathrm{CAP})(\mathrm{CAPC})$

CAPC: capacity cost in dollars per $\mathrm{KW}$; an input parameter

Capital costs are also a function of the regulated return to equity (ER), the bond rate (BR), the debt to equity ratio, and deprectation (DEPR ${ }_{t}$ ).
(2) $E=(E K)(K)(E R)$, equity costs,
(3) $D=(D K)(K)(B R)$, debt costs,
(4) $D_{E P R_{t}}=\left(\mathrm{DEP}_{t}\right)(\mathrm{K})$, depreciation.

and therefore

(5) $\mathrm{CC}_{t}=\mathrm{E}+\mathrm{D}+\mathrm{DEPR}_{\mathrm{t}}$ 
where, EK: equity share of capital; an input parameter,

DK: debt share of capital; an Input parameter

$$
(E R=1-D K)
$$

$\mathrm{DEP}_{t}$ : depreclation rate in year $t$;

(6) $\operatorname{DEP}_{t}:(T+1-t) / \sum_{t=1}^{T} t$,

where $T$ is the length of time for electricity production. The model provides fast

for a write-off option.

Operating costs are a function of total investment and escalate through time.

(7) $O M C_{t}=\left(O C_{t}\right)(K)$

(8) $O C_{t}=(O C B)(1+\dot{O C})^{t}$

$O C_{t}$ : operating percent of Investment in year $t$; input variable

$O C B$ : base rate; input variable,

oc: escalation rate; input variable

Likewise, property taxes are a function of investment.

(9) $\quad$ PTX $=($ PTP $)(K)$.

PTP: property tax rate; input variable.

Before income taxes are calculated, total revenues must be known.

They are, of course, a function of the price of electricity $\left(\mathrm{PE}_{t}\right)$ and the quantity of electricity $(Q E)$, which is a function of plant capacity. $P E_{t}$ is specified as an exogenous busbar price of electrictity which is determined by the market and escalates over time.

(10) $P E_{t}^{t}=(P B)(1+\dot{P})^{t}$

PB: Base busbar price of electricity; input variable.

$\dot{P}$ : escalation factor; input variable. 


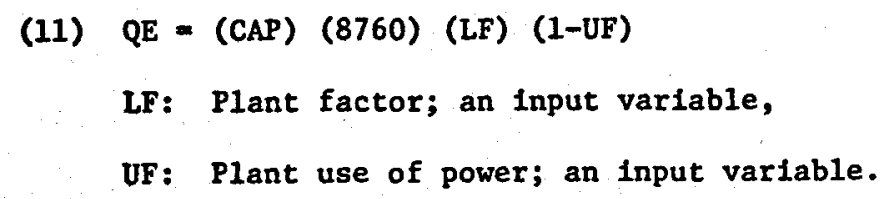

Income taxes and energy costs must be determined simultaneously, because the price of the geothermal energy $\left(\mathrm{PHW}_{t}\right)$ is an element of both and moreover, PHW $_{t}$ is the output of the utility sector for the model. The quantity of geothermal energy is determined by electricity plant requirements.

$$
\begin{aligned}
& \text { (12) } \mathrm{QHW}=(\mathrm{CAP})(8760)(\mathrm{LF}) \\
& \text { (13) } \mathrm{ENC}_{\mathrm{t}}=\left(\mathrm{PHW}_{\mathrm{t}}\right)(\mathrm{QHW}) \\
& \text { (14) } \operatorname{ITX}_{t}=\{\operatorname{TXRT}\}\left\{\left[\left(P E_{t}\right)(Q E)\right]-\left[O M C_{t}\right]-[P T X]-\left[E_{t}\right]\right. \\
& \left.-\left[\operatorname{DEPR}_{t}\right]\right\}-\left\{[\mathrm{CRRT}]\left[\mathrm{K}_{t=1, t=2, t=3}\right]\right\} \\
& \text { TXRT: Income tax rate; input variable } \\
& \text { CRRT: investment tax credit rate; input variable }
\end{aligned}
$$

Therefore,

$$
\text { (15) } \begin{aligned}
\mathrm{PHW}_{t}= & \frac{\left(\operatorname{TXRT}_{\mathrm{N}}\left(\mathrm{DEPR}_{t}\right)+(\mathrm{CRRT})\left(\mathrm{K}_{t=1, t=2, t=3,}\right)-\left(\mathrm{CC}_{t}\right)\right.}{(\mathrm{QHW})(1-\mathrm{TXRT})} \\
& +\frac{\left(\mathrm{PE}_{t}\right)(\mathrm{QE})-\left(\mathrm{OMC}_{t}\right)-(\mathrm{PTX})}{(\mathrm{QHW})}
\end{aligned}
$$

The price of energy in year ( $t$ ) from (15) and the quantity of energy from (12), that is, ENC (equation 13), become total revenue for the geothermal energy producer.

\section{Geothermal Energy Producer Sector}

We will recall equation (13) $\mathrm{ENC}_{t}$ for the producer, $\mathrm{REV}_{f}$, or total revenues. OR,

$$
\text { (16) } \mathrm{REV}_{\mathrm{j}}=\mathrm{ENC}_{\mathbf{t}}
$$

First, a discussion of the time period involved is necessary. The producer's time horizon is different from that of the electric utility. The years $t$ are elements of $T$, while the years $j$ are elements of $J . J-T=P P Y$, the 
"pre-production years". PPY is composed of two periods. The exploration period (EXP) and the development period (DVP), and thus EXP+DVP=PPY. Thus the determinative direction is EXP+DVP+T=J. EXP is the period during which only exploratory wells are drilled. The development period begins with the drilling of the first production well.

The producer's activity is modeled somewhat differently from the utilities because the output of the simulation is a rate of return on the producer's equity. Thus, the first order of business is to determine what the level of the investment is in each year of the project. The producer's investment $\left(\mathrm{PNV}_{j}\right)$ is a share of the total investment in year $j\left(\operatorname{PPK}_{j}\right)$.

$$
\begin{aligned}
& \text { (17) } P N V_{j}=(E P K)\left(P K K_{j}\right) \\
& \text { (18) } \operatorname{PKK}_{j}=\left(\mathrm{LNV}_{j}\right)+\left(\mathrm{DNV}_{j}\right)+\left(\mathrm{IDC}_{j}\right)+(\mathrm{OPNV}) \\
& \text { EPK : Equity portion of capital; an input variable, } \\
& \mathrm{LNV}_{j} \text { : Leasing investment in year } j \text {, in } \$ \text {, } \\
& \text { DNV }_{j} \text { : Drilling investment in year } j \text {, in } s \text {, } \\
& I_{j} \text { : Interest Cost during Construction in year } j \text {, in } \$ \text {, } \\
& \text { OPNV }_{j} \text { : Other producer investments in year } j \text {, in } \$ \text {, }
\end{aligned}
$$

Total Investment by the producer, cumulative to year $j$ is

$$
\text { (19) } \mathrm{PK}_{j}=\sum_{i=0}^{j} \mathrm{PKK}_{i}
$$

We will first describe the calculation of each element of PKK $j$, beginning with $D_{j}{ }_{j}$ 
(20) $\left.\mathrm{DNV}_{j}=\left[\frac{\mathrm{NPW}_{j}}{\mathrm{PSS}}\right)(\mathrm{ADDP})(\mathrm{CPFP})\right]+\left[\left(\frac{\mathrm{NIW}_{\mathfrak{j}}}{\mathrm{ISS}}\right)(\mathrm{ADDI})(\mathrm{CPFI})\right]$

$\mathrm{NPW}_{f}$ : New producing wells in year $j$,

PSS: Production Well Success ratio,

CPFP: Cost per foot, producing wells,

$N W_{j}$ : New injection wells in year $j$,

ISS: Injection well success ratio ( $=1$ usually),

ADDI: Average depth of injection wells,

CPFI: Cost per foot of injection wells,.

The calculation of the elements of $\mathrm{DNV}_{j}$ is shown below.

(21) $\mathrm{NPW}_{j}=R P W_{j}-R P W_{j-1}$

$R P W_{j}$ : required producing wells, $R P W_{j}=0$ when $j<E X P+1$.

(22) $\mathrm{RPW}_{\mathrm{j}}=\frac{{ }^{\mathrm{RFR}} \mathrm{CAP}}{\mathrm{FR}}$

FR: flowrate in $1 \mathrm{~b} / \mathrm{hr}$ per producing well,

${ }^{R F R}$ CAP : required flowrate for rated capacity in $\mathrm{lb} / \mathrm{hr}$ (fn) $^{(\mathrm{f})}$

(CAP is stated here in MW, not KW, and is net of UF.)

(23a) $\mathrm{RFR}_{50}=1374.912 \mathrm{e}^{-0.01379 \text { (TEMP) }}$

(23b) $\mathrm{RFR}_{100}=2667.012 \mathrm{e}^{-0.01372 \text { (TEMP) }}$

(23c) $\mathrm{RFR}_{150}=3959.226 \mathrm{e}^{-0.013655 \text { (TEMP) }}$

(23d) $\mathrm{RFR}_{200}=5251.44 \mathrm{e}^{-0.01359(\mathrm{TEMP})}$

Bechtel Corporatern, Alvoucad Design and Economic

considerations for Commercial Geoperneal Pever Plaxly at Hcten and Niland, Californea. SAN-1124-2, Sam Francesco, Ca 1977 
Temperature decline is estimated, and resulting need for increased flow rate, thus producing wells, is modeled in the following way:

$$
\begin{aligned}
& \text { (24a) Temp }=\frac{T_{0}}{360}\left[358.344 \mathrm{e}^{-0.00144(\mathrm{Time})}\right] \text {, for CAP }=50 \mathrm{MW} \text {. } \\
& \text { (24b) Temp }=\frac{T_{0}}{360}\left[357.474 \mathrm{e}^{-0.00233(\text { Time) }}\right] \text {, for CAP }=100 \mathrm{MW} \\
& \text { (24c) Temp }=\frac{T_{0}}{360}\left[357.12 e^{-0.00322(\text { Time })}\right] \text {, for } C A P=150 \mathrm{MW} \\
& \text { (24d) Temp }=\frac{T_{0}}{360}\left[357.769 \mathrm{e}^{-0.00411(\mathrm{Time})}\right] \text {, for } \mathrm{CAP}=200 \mathrm{MW} \\
& \text { downhole } \\
& T_{0} \text { : Intial temperature of brine in }{ }^{\circ} \mathrm{F} \text {, an input variable. } \\
& \text { (25) } \mathrm{NIW}_{\mathrm{j}}=R I W_{j}-R I W_{j-1} \\
& \mathrm{RIW}_{j} \text { : required injection wells in year } f \text {. } \\
& \text { (26) } \mathrm{RIW}_{\mathrm{J}}=\mathrm{RPW}_{\mathrm{J}}(\mathrm{PIR})^{-1} \\
& \text { PIR: required ratio of producing wells to injection wells, } \\
& \text { in year } j \text {, an input variable. }
\end{aligned}
$$

After Initiation of power generation, $\mathrm{NPW}_{j}$ and $\mathrm{NIW}_{j}$ will generally take on a value of 0 or 1 , but are arbitrarily allocated during DVP.

The remaining elements of $\mathrm{PKK}_{\mathbf{j}}$ are discussed below:

(27) $\mathrm{LNV}_{f}=\left[(\right.$ DPAC $\left.)(\mathrm{ACPW})\left(\mathrm{NPW}_{j}\right)\right]+\left[(\right.$ DPAC $\left.)(\mathrm{ACIW})\left(\mathrm{NIW}_{j}\right)\right]$

DPAC: dollars per acre leasing cost; input variable, ACPW: acres per producing well; input variable, ACIW: acres per injection well; input variable. 
(28) $\mathrm{OPNV}_{j}=\operatorname{EXPN}_{j}+\mathrm{PIPN}_{j}+\mathrm{ONV}_{j}$

$\operatorname{EXPN}_{j}$ : exploration investments, year $j$,

PIPN $_{j}$ : plping investments, year $j$,

ONv ${ }_{j}$ other investments, year $j$.

(29) $\operatorname{EXPN}_{j}=f(E X P N)$

(30) $\operatorname{EXPN}=($ EXD) $($ CAP $)$

EXD: dollars per unit of capacity (electric) needed for exploration, on average; input variable.

$j>\operatorname{EXP}, \operatorname{EXPN}_{j}=0$

EXPN; total exploration investment in \$

(Note: $\operatorname{EXPN}=\sum_{j=0}^{\operatorname{EXP}} \operatorname{EXPN}_{j}$, where the distribution function is chosen arbitrarily)

(31) $\operatorname{PIPN}_{j}=(P I P)\left(D N V_{j}\right)$

PIP: average percentage of drilling investment required for piping and similar field developments.

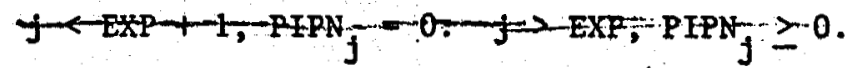

(32) $O N V_{j}=\left(\mathrm{OPE}_{j}\right)\left(\mathrm{DNV}_{j}\right)$

(33) $O P E_{j}=(O P E B)(1+O P)^{j}$

Equations (32) and (33) calculate estimate additional investments in

field development which are related to drilling expenses. 
(34) $\mathrm{IDC}_{j}=[\mathrm{BR}][\mathrm{DPK}]\left[\sum_{1=0}^{j} \mathrm{LNV}_{1}+\sum_{1=0}^{j} \mathrm{DNV}_{1}+\sum_{1=0}^{j} \mathrm{OPNV}_{1}\right]$

$I D C_{j}=0$ for $j>$ PPY.

DPK: debt portion of capital; DPK $=1-$ EPK.

Thus, total revenues and all elements of investment have been calculated. What remain are uncapitalized elements of cost. $\mathrm{OMCP}_{j}$ are operating and other current outlays.

(35) $\mathrm{OMCP}_{j}=\left[\left(\mathrm{OCP}_{j}\right)\left(\mathrm{PK}_{j}\right)\right]+\left[(\mathrm{BR})\left(\mathrm{PK}_{j}\right)(\mathrm{DPK})\right]+\left[\mathrm{PTXP}_{j}\right]$

(36) $\mathrm{OCP}_{j}=(\mathrm{OCPB})\left(1+O \mathrm{CP}^{j}\right)^{j}$

$\mathrm{OCP}_{j}$ : operating percentage of investment outlay,

OCPB: base percentage; input varfable,

oćp: escalation factor.

$\operatorname{PTXP}_{\mathbf{j}}:$ property taxes.

(37) $\operatorname{PTXP}_{j}=(\operatorname{PTPP})\left(\mathrm{PK}_{j}\right)$

PTPP: property tax rate; input variable.

Income taxes are as follows:

(38) $\operatorname{ITXP}_{j}=\{\operatorname{TXRT}\}\left\{\left[\left(\operatorname{REV}_{j}\right)(1-\lambda)(1-Z)\right]-\left[\left(\operatorname{OMCP}_{j}\right)-(B R)\left(P K_{j}\right)(D P K)\right]\right.$

$-\left[(Y)\left(\right.\right.$ DNVD $\left.\left.\left._{j}\right)\right]\right\}-\left\{[\operatorname{CRRT}]\left[(1-Y)\left(\sum_{\substack{j=j-2 \\ j}}^{j} D_{1}\right]\right\}\right.$

(39) $\operatorname{DNVD}_{j}=\sum_{i=1}^{j} \operatorname{DNV}_{i}\left(\operatorname{DEPRP}_{j-i+1}\right)^{\prime}$ 


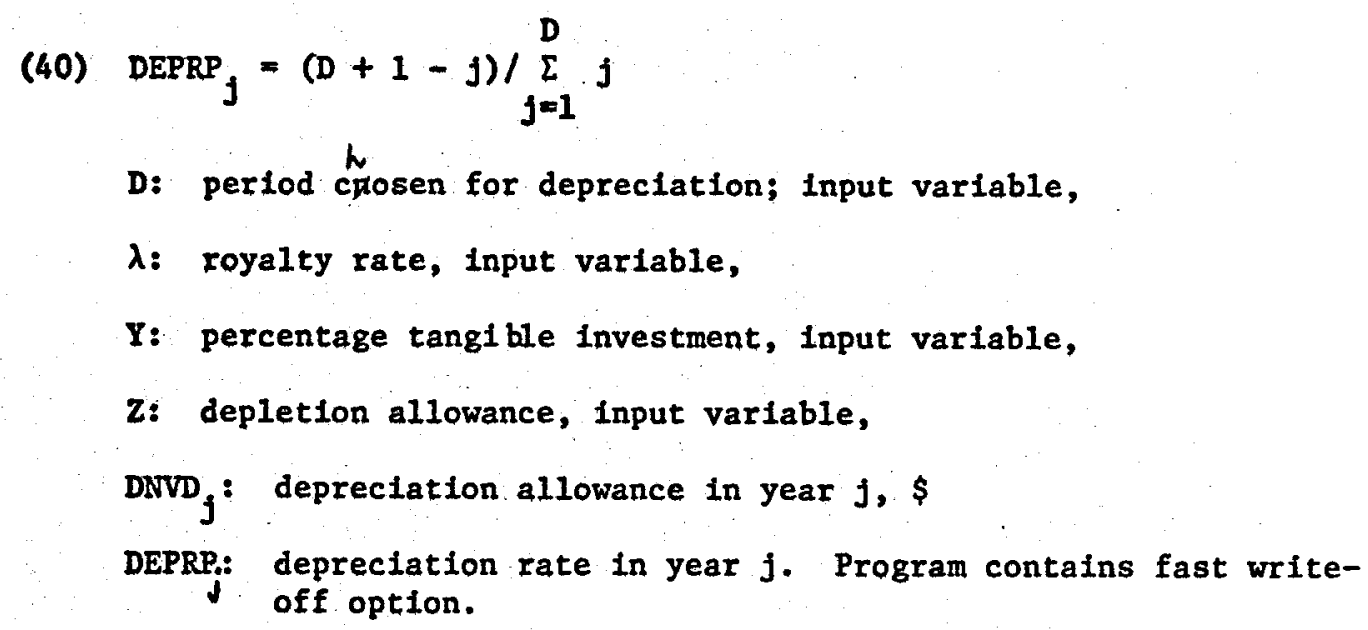

Finally, the model solves for $R$, the rate of return to equity for the geothermal energy producer from the Jth degree polynomial given by

$$
\text { (42) } \sum_{j=0}^{J}\left\{\left[\left(\operatorname{REV}_{j}\right)(1-\lambda)-\left(\operatorname{OMCP}_{j}\right)-\left(\operatorname{ITXP}_{J}\right)\right]\right\} e^{-R j}=0
$$

The $\mathrm{R}$ thus solved is one criterion for ranking geothermal resource sites and for comparing the outcomes of policy variable changes. 


\section{DISCUSSION OF}

PACIFIC GAS AND ELECTRIC COMPANY

COST OF POWER ANALYSIS PROCEDURES

The intent of PGandE economic analysis is to give our management realistic information about probable total costs of available engineering alternatives. We, therefore, attempt to include all future cost impacts of such alternatives. Given the large number of variables involved and the need to make the results of the analysis understandable we find comparison of Level Annual Revenue Requirements the most appropriate economic analysis procedure.

Generation plant capital costs are developed by the Engineering Department, broken down by California Public Utility Commission accounts. Estimates of plant engineering, construction supervision and corporate overheads, escalation, AFDC and ad valorim taxes during construction are provided by our Economics and Statistics Department.

The Economics and Statistics Department also provides fixed charge rates for each capital account which are used to convert plant capital costs to level annual revenue requirements. The components of these fixed charge rates include the Company's weighted cost of capital, salvage forecasts, retirement dispersion effects on capital recovery, state and federal income taxes and insurance.

Operating and maintenance expense forecasts are made by the Operating Department. Fuel cost estimates are obtained from a variety of sources. Nuclear fuel cycle cost analysis is performed by the Engineering Department. Coal costs are developed by the Coal Resources Department. Oil and natural gas cost forecasts are made by the Materials Department. Geothermal energy costs are based on a contractual relationship of actual fossil and nuclear plant operating costs so forecasts of future costs are developed from detailed production cost analysis'.

Basic cost escalation forecasts are made by the Economics and Statistics Department based on analysis of data from various national economic forecasting services.

Using the data discussed above the Engineering Planning Department performs several types of economic analysis. Generic analysis of generation alternatives provides cost of power comparisons for different generating technologies at various average annual capacity factors. Production cost analysis forecasts monthly production costs for alternative long-range resource plans. Site specific analysis gives management and regulatory agencies detailed cost of power estimates for proposed generation projects. 
PGandE uses its cost of capital as the discount rate used in levelizing and present worth calculations. Economic results are expressed in both current and constant dollar terms.

J. H. Malinowski

June 29,1978 
PGEE FOSSIL I 2

COST OF POWER CALCULATION

$1600 \mathrm{MW}$ PLANT

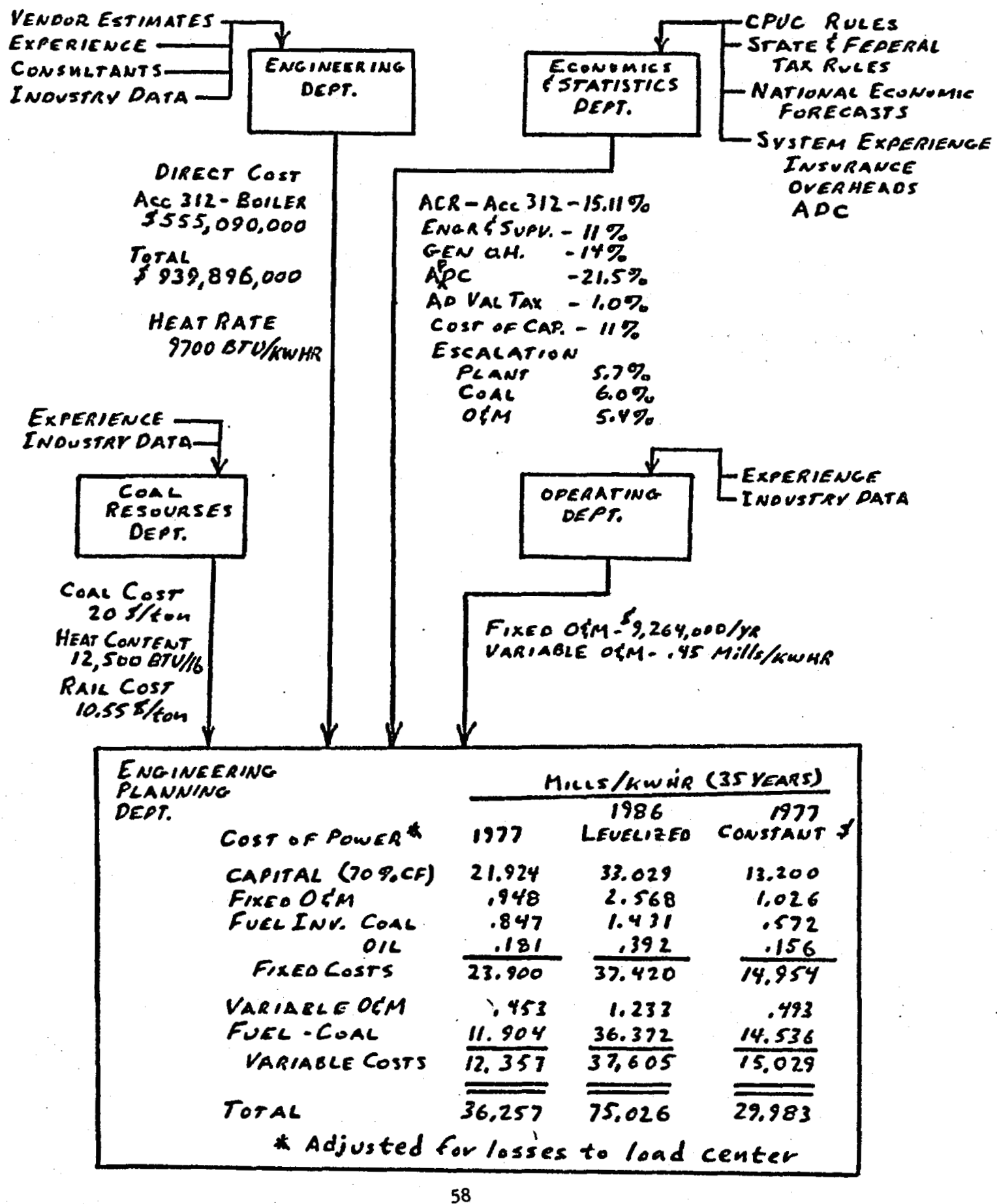


Discussion of Levelizing and

Constant Dollar Economic Analys is

Comparison of Level Annual Revenue Requircments (IARR) of alternatives is the generally accepted method of utility economic analysis. The L $\Lambda \mathrm{RR}$ of a project includes the economic effect of all expected expenditures associated vith the project. Constant Dollar Analysis is a useful and equivalent version of IARK analysis.

Levelizing procedures are key elements of the IARR method and thereforc of utility economic analysis. The levelized value of a time varying series of expenditures is the constant (or level) series of expenditures with the same present worth as the tirue varying series. In mathematical form, this statement is:

$$
\sum_{k=0}^{n-1} \frac{L_{e}}{(1+i)^{k}}=\sum_{k=0}^{n-1} \frac{E_{k}}{(1+i)^{k}} \Longrightarrow L_{e}=\frac{\sum_{k=0}^{n-1} \frac{E_{k}}{(1+i)^{k}}}{\sum_{k=0}^{n=1} \frac{1}{(1+i)^{k}}}
$$

where $L e$ is the levelized value of the series $E_{k}$

$n$ is the number of time periods considered

$i$ is the cost of capital

$E_{k}$ is the kth expenditure of the time-varying series and should include expected escalation.

While comparison of the levelized cost of alternatives correctly considers the economic impact of all known future expenditures, the levelized costs include the effect of today's high inflation in both expenditures ard cost of capital. The actual and levelized expenditures are both defined as current (escalated) dollar expenditures. In periods of sustained general inflation the value of current dollars decline with time in real terms. The effects of general inflation can be eliminated by evaluating alternatives in constant dollar terms. This does not eliminate the effect of real price changes. Constant dollars maintain their real value by escalating at the general inflation rate in current dollar terms. The process of levelizing in constant dollar tirms is:

$$
\sum_{k=0}^{n-1}\left(\frac{1+g}{1+i}\right)^{k} L_{c}=\sum_{k=0}^{n-1} \frac{E_{k}}{(1+i)^{k}} \Longrightarrow L_{c}=\frac{\sum_{k=0}^{n-1} \frac{E_{k}}{(1+i)^{k}}}{\sum_{k=0}^{n-1}\left(\frac{1+2}{1+i}\right)^{k}}
$$

where $\quad L_{C}=$ constant dollar levelized value of the series Ek

9 the general escalation or inflation rate

$i, n, E_{K}$ as previously defined 
Levelized current dollar and levelized constant dollar expenditures are directly proportional,

$$
L_{c}=K_{n} L_{e} \text { where } K_{n}=\frac{\sum_{k=0}^{n-1} \frac{1}{(1+i)^{K}}}{\sum_{k=0}^{n-1}\left(\frac{1+0}{1+6}\right)^{K}}
$$

and these two economic measures are equivalent since $K_{n}$ is indcpendent of any altcrnative specific parameter, (i.e., it is only dependent on life, $n$, discount rate, $i$, and general inflation rate, $g$ ).

Kn can be gencralized to include $m=$ the number of years between the constant dollar year and the initial operation year. The resulting equation becomes:

$$
K_{m, n}=\frac{1}{(1+g)^{m}} \frac{\sum_{k=0}^{n-1} \frac{1}{(1+i)^{k}}}{\sum_{k=0}^{n-1}\left(\frac{1+g}{1+j}\right)^{k}}
$$

Constant dollar analysis will result in the same economic choices as current dollar analysis. Constant dollar costs, however, are more casily compared to present-day costs since general inflationary effects are factored out.

Attachments

1. Sample calculations

2. Detailed discussion of constant dollar analysis 


\section{SAMPLE CAI.CUR.ATIONS}

Cost of power estimates for Fossil 1 \& 2 illustrate the resulis of levelized current dollar and levellzed constant dollar analysis. For the Butte site, the cost of power components with no escalation assumed are:

\section{Cost of Yower Component}

Capital

O\&M Fixed

Coal Inventory

Oil Inventory

Total Fixed Cost (70\% C.F.)

Osti Variable

Fuel ( $\operatorname{Cos} 1)$

Total Cost of Power

\section{Mi11s/Kwhr}

20.946

.967

.890

.357

23.160

.453

11.908

35.521

We do not consider this a valid economic measure since it includes inflationary impacts in the cost of capital, but not other cost of power components.

The escalation assumptions currently used for resource alternative cconomic analysis include:

$\begin{array}{ll}\text { Goneral Inflation Rate } & 5 \% \\ \text { Steam Cycle Plant Escalation Rate } & 5.7 \% \\ \text { Coal Fuel Cost Escalation Rate } & 6.0 \% \\ \text { Oil Fuel Cost Escalation Rate } & 10 \% \text { to } 1983 \\ & 7 \% \text { beyond } \\ \text { O\&M Escalation } & 5.4 \%\end{array}$

Using these escalation assumptions and a 35-year econoinic life results in the following escalation factors for conl plants operational in 1980 , 1985 and 1990.

\section{Current Dollar Escalation Factors}

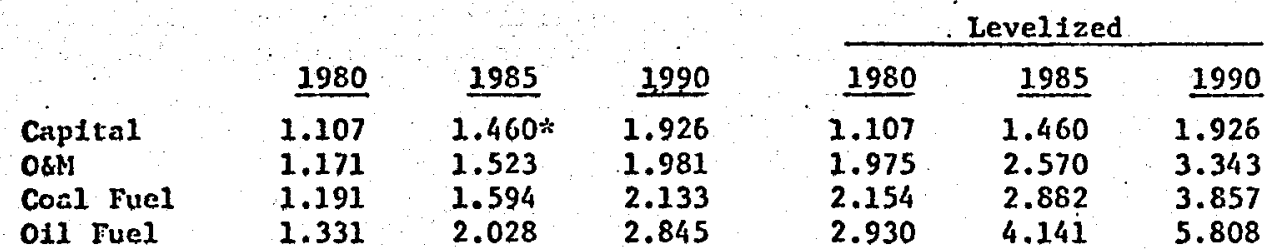

* GM escalation assumed.

The 1977 constant dollar multipliers $(\mathrm{Km}, \mathrm{n})$ for these start dates and a 35-year life are:

Start Date
1980
1985
1990

$\frac{\text { Multiplier }}{.536}$


Applying these multipliers to the current dollar escalation factors yield 1977 constant dollar escalation factors.

1977 Levelizcd Constant Dol.1ar Escalition Factors

$\begin{array}{llll} & 1980 & 1.985 & 1990 \\ \text { Capital } & .593 & .613 & .634 \\ \text { OsN } & 1.058 & 1.078 & 1.100 \\ \text { Coal Fuel } & 1.154 & 1.210 & 1.268 \\ \text { Oil Fuel } & 1.569 & 1.739 & 1.910\end{array}$

Using the unescalated cost of pover components and these escalation factors results in the following current dollar and constant dollar levelized cost of power estimates:

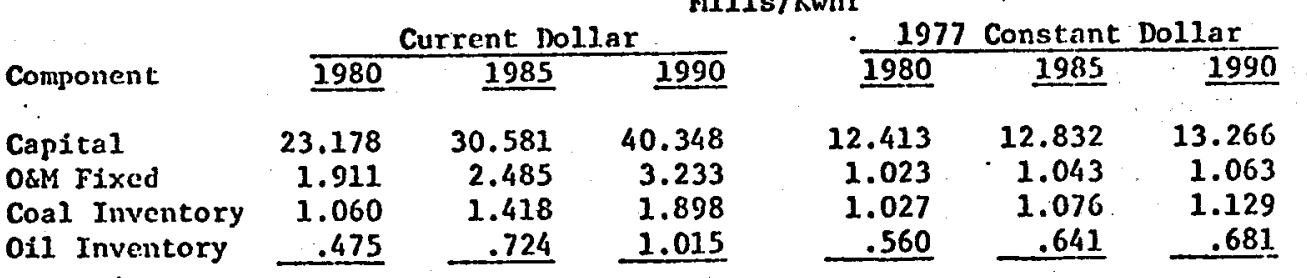

Total Fixed

$\begin{array}{lrrrrrr}\text { O\&N Variable } & .900 & 1.170 & 1.522 & .482 & .491 & .500 \\ \text { Fucl (Coal) } & 25.649 & \underline{34.324} & \underline{45.933} & \underline{13.736} & \underline{14.403} & \underline{15.102}\end{array}$

Total cost

of Power

\section{$53.172 \quad 70.702 \quad 93.949 \quad 29.241 \quad 30.487 \quad 31.741$}

A coal plant built in 1990 will produce power at a levelized current dollar cost $77 \%$ highar than the same plant built in 1900 . The difference in 1977 constant dollar costs is $8.5 \%$ reflecting real escalation of plant and operating costs. 
CENERALLEE PONER PRODUCTION COSTS TOR VAR IOUS TYPES OF GENERATIIG FACILITLES

\begin{tabular}{|c|c|c|c|c|c|}
\hline & & & Combined & & \\
\hline $\begin{array}{l}\text { Nuelear } \\
\text { xi200 }\end{array}$ & $\begin{array}{r}7 C D \\
2 \times 800 \mathrm{MN} \\
\end{array}$ & $\begin{array}{l}\text { W/O FCD } \\
2 \times 800 \mathrm{MW}\end{array}$ & $\begin{array}{l}\text { Cycle } \\
400 \mathrm{in} \\
\end{array}$ & $\begin{array}{l}\text { Turbine } \\
4 \times 75 \mathrm{MW}\end{array}$ & $\begin{array}{c}\text { Geothersal } \\
110 \mathrm{~ms}\end{array}$ \\
\hline
\end{tabular}

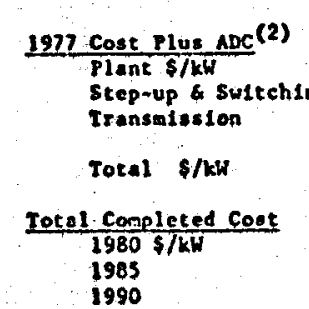

Fixed Charge Rates

Fuel Type

Juel Inventory (Days)

Average Heat Iate

Osy Cost Flxed (\$/kWyz)

Veriable (Mille/khh)

Peak Transutaton Loes $z$ Average

35 Year Levelized Power Production Coste for Plants coupleted in 1985

$$
\begin{aligned}
& \text { Fixed } \$ / \text { kWy } \\
& \text { thWhr } 9 \text { 7orcr } \\
& \text { variable t/kWhr }
\end{aligned}
$$

Total

35 Year levelized Cost:

on .1977 Constant $\$$ Bas (3)

$$
\text { thihr, } 10 \mathrm{CZ} \text { CF }
$$

$$
\begin{aligned}
& 102 \\
& 30 \\
& 50 \\
& 60 \\
& 70 \\
& 60
\end{aligned}
$$

$\begin{array}{rr}43 & 7 \\ 20 & \\ 30 & \\ 893 & 791 \\ & \\ 878 & 804 \\ 1159 & 1061 \\ 1529 & 1400\end{array}$

15.9

Urantum \begin{tabular}{rr}
761 & 544 \\
20 & 20 \\
\hline
\end{tabular}

10

791

804
1061
1400

25.4

Coal

Conl
264
13
10

10

289

320

412
541

15.5

Dietilete

122
145
194
260
466

263
370
530
743
1462

90.

$\approx 700$

10,300 .

2.8

9,700

9,230

\begin{abstract}
5.8
.5
\end{abstract}
4.3
.1

8,600

5.0
.4

1.4
.1

-.4
.5

2.0
1.9

-2.0
. .7

201
3.3
2.1

$\begin{array}{r}193 \\ 3.2 \\ 3.6 \\ \hline\end{array}$

147
2.4

$\begin{array}{r}2.4 \\ 3.3 \\ \hline\end{array}$

90

5

6.8

- 5.7

$\begin{array}{r}1.5 \\ 9.4 \\ \hline\end{array}$

10.9

$\begin{array}{r}4.7 \\ 12.6 \\ \hline\end{array}$

67

$\begin{array}{r}1.4 \\ 2.9 \\ \hline\end{array}$

13.3

4.3

(1) rGD - Flue Gas Desulfurization (Scrubbera)

(2) ADC - Allowance for Funds During Construction

(3) Cost with effects of zeneral escalation factored out 
GENEPALIZED PONER PRODUCTION COST ESTTMATES FOR VARIOUS FUTURE DISPATCHABLE GLVEPATIOA TYPES

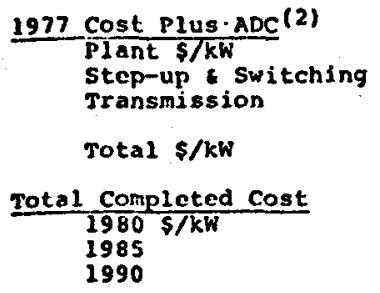

Fixed Charge Rates

Fuel Type

\begin{tabular}{ll} 
Fuel cost $4 / M M B T U$ \\
\hline 1977 \\
1980 \\
1985 \\
1990 \\
2000
\end{tabular}

Fucl Inventory (Days)

Average Heat Rate (Btu/kWhr.)

O\&M cost Fixed ( $\left.\$ / k W y r_{.}\right)$

$$
\text { Variable (Milis/kwh) }
$$

Peak Transmission Loss

Average

35 Year Levelized Power Production

costs for Plants completed in 1985

Fixed $\$ / k$ Wyr

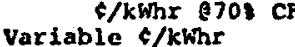

Total

1977 Constant \$ Levelized

costs $(3) \& / k$ Whr $108 \mathrm{CF}$

.
Intergrated Coal Gasifler

combined

Crele(1)

$\begin{array}{r}782 \\ 20 \\ 30 \\ \hline\end{array}$

B31

844

2115
1471

25.0

Coal

122

145

194

260
466

90

7,900

5.8
.5

2.0
1.9

192

192
3.1
3.0

6.2

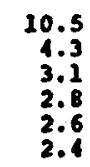

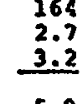

5.9

Fuel

Cel1 (1)

$\begin{array}{r}656 \\ 26 \\ \hline\end{array}$

$\begin{array}{r}20 \\ -15 \\ \hline\end{array}$

691

703

927
1223

25.4

coal

125

199

267

477

90

8,500

5.8
.5

-2.0
-.7

9.2
4.0
2.9
2.6
2.5
2.3
Blomasa

Direct

Combustion

Geothermal

Inquia Dominated (1)

$$
\begin{array}{r}
971 \\
15 \\
35 \\
\hline
\end{array}
$$

1021

$$
\begin{array}{r}
589 \\
15 \\
100 \\
\hline
\end{array}
$$

704

$\begin{array}{lr}1128 & 778 \\ 1488 & 1026\end{array}$

$1964 \quad 2354$

15.4

15.0

Biomas

Hot Water

$\begin{array}{rr}125 & 72 \\ 149 & 85 \\ 199 & 114 \\ 267 & 253 \\ 477 & 273 \\ 60 & - \\ 6.000 & 30,000 \\ 23.3 & 9.2 \\ 3.8 & 1.5 \\ 3.8 & 7.3 \\ 3.5 & 6.7\end{array}$

309

309
7.0
7.0

190

7.0

$\begin{array}{r}3.8 \\ \hline\end{array}$

12.1

7.9

17.7
7.9
5.9
5.4
5.2
4.8

12.2

12.2
5.1

3.8

3.5

3.3

(1) This technology is not commercially avallable today.

(2) $A D C=$ Allowance for Funds During construction.

(3) Cost with effects of general inflation factored out.

In the absencs of proven or commercially avallable technology, many assumptions regarding performalce characteristics and costs must be made. Information currenty available produces a wide range of possible assumptions. Those used for these cstimates are neitier the most optimistic nor the most pessimistic. The accompanying: data and explanation discusses the assumptions used. These estimates are crovided without regard to when the resource may be comercially avaliable, whether it would be suitable for a jaseload resource without backup capacity or the relative rellability of the resource.

- ful cuts $=50 \%$ more than feysass. 
GENERALI2ED POWER PRODUCTION COST ESTIMATES

FOR VARIOUS SODAR GENERATION TYPES (I

1977 Cost Plus ADC (2)

Plant $\$ / \mathrm{k}$.

step-up 4 switching

Transmission

Total $\$ / k W$

$\frac{\text { rotal Completed cost }}{1980 \$ 7 \mathrm{kh}}$

1985

Fixed Charge Rate

Energy Source

Supplemental Fuel cost $4 /$ MuTU 2977

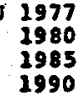

2985

2000

GT ruel Inventory Days

GT heat Rate

solar system CP

O\&N Cost rixed $\$ / k w y x$ GT Varlable Hilis/kWh

Solar System Peak Transmlssion

Losses

Aver age (4)

35 Year Levelized Power Production costs for Plants Completed In 1985

Fixcd $\$ / \mathrm{kWyr}$

Variable GT Energy 108 CF Operation

rotal $\$ /$ kWhr

1977 Constant \$ Levellzed costs (5)

GT $\mathrm{CF} O$
Fual

Saving

1622

100

1741

$1924 \quad 2108$

$\begin{array}{ll}2538 & 2769 \\ 3349 & 3651\end{array}$

15.3

15.3

Sun Sun plus

Sun Plus
Distillate

$\begin{array}{rr}-\quad & 263 \\ -\quad & 370 \\ - & 743 \\ - & 2462\end{array}$

$-\quad 10$

$-11,500$

$40 \quad 10$

$\begin{array}{rr}10.5 & 20.9 \\ 0 & 1.6\end{array}$

$\begin{array}{ll}7.3 & 7.3 \\ 6.7 & 6.7\end{array}$

\begin{tabular}{rr}
445 & 487 \\
22.7 & 12.2 \\
- & 2.6 \\
\hline
\end{tabular}

13.7
Solar Photovoltalc

Fuel

saving

GT

back-up (3)

2075
20
100

100

2227
33

100

2360

24252550

$3200 \quad 3431$

$4222 \quad 4524$

25.3

15.3 .

Bun sun Plus

Dlstillate

$\begin{array}{rr}- & 263 \\ = & 370 \\ - & 530 \\ - & 743 \\ - & 1462\end{array}$

- 5

$-\quad 11,500$

20

$8: 8$

20

9.2
1.6

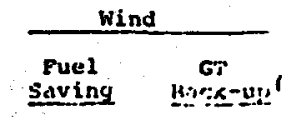

\begin{tabular}{rr}
762 & 914 \\
0 & 13 \\
50 & 50 \\
\hline 812 & 977
\end{tabular}

$510 \quad 209$

$1200 \quad 143$

$1584 \quad 1886$

15.3

15.3

wind wind plu.

Distiliat

- $2 ;$

- $\quad 530$

743
$-\quad 1462$

- 10

- 11,500

$35 \quad 35$

$\begin{array}{ll}3.1 & 3.5 \\ 0 & 1.6\end{array}$

7.37 .3

$\begin{array}{ll}3.8 & 3.8 \\ 3.5 & 3.5\end{array}$

5.3
-
$=$
$=$

5.8
5.8
5.7
5.7
5.7

$6.7 \quad 6.7$

\begin{tabular}{rr}
549 & $\begin{array}{r}590 \\
31.3\end{array} \quad \begin{array}{r}26.9 \\
2.6\end{array}$ \\
\hline 31.3 & 29.5
\end{tabular}

\begin{tabular}{ll}
198 & 239 \\
6.5 & 6.3 \\
$-\quad$ & 2.8 \\
\hline 6.5 & 8.9
\end{tabular}

$\begin{array}{ll}2.7 & 3.3 \\ = & 3.5 \\ = & 3.7 \\ - & 3.9\end{array}$

(1) These technologles are not comercially avallable today.

(2) $A D C=$ Allowance for Funds buring Construction.

(3) This anaiyais assumes 13 of the solar resource capacity is firw. Gas turblne back-up capacity of $85 \mathrm{t}$ is assumed.

(i) Gas turbine losses are assumed to be -5.71 peak, -2.21 average

(5) Costs with effects of general inflation factored out.

In the absence of proven or comerclally avallable tochnology, many asumptions regarding perforsacharacterlstics and costs must be made. Information curreitly avaliable produces a wide range of poss;: characterlstics and costs nust be made,

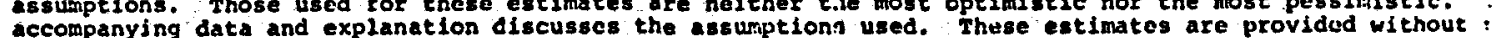

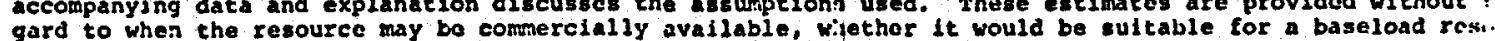
witlout backup capacity or tho rolative reliability of tho roaurco. 
CAPITAL COST, REVENUE REQUIREMENTS AND NET SALVAGE FOR THE BUTTE SITE

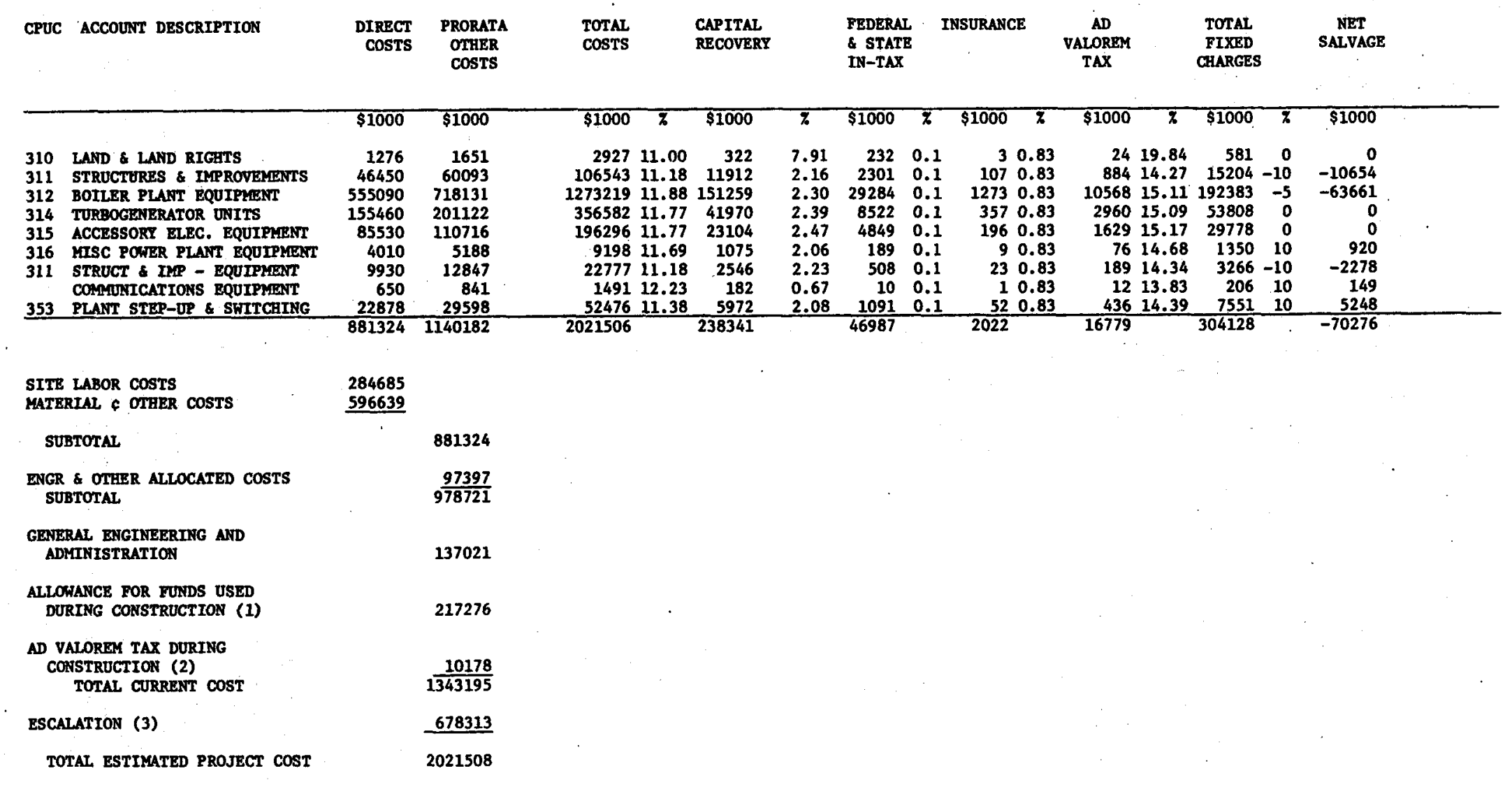


CAPITAL COST, REVENUE REQUIRMTENS AND NET SALVAGE FOR THE BUTTE SITE

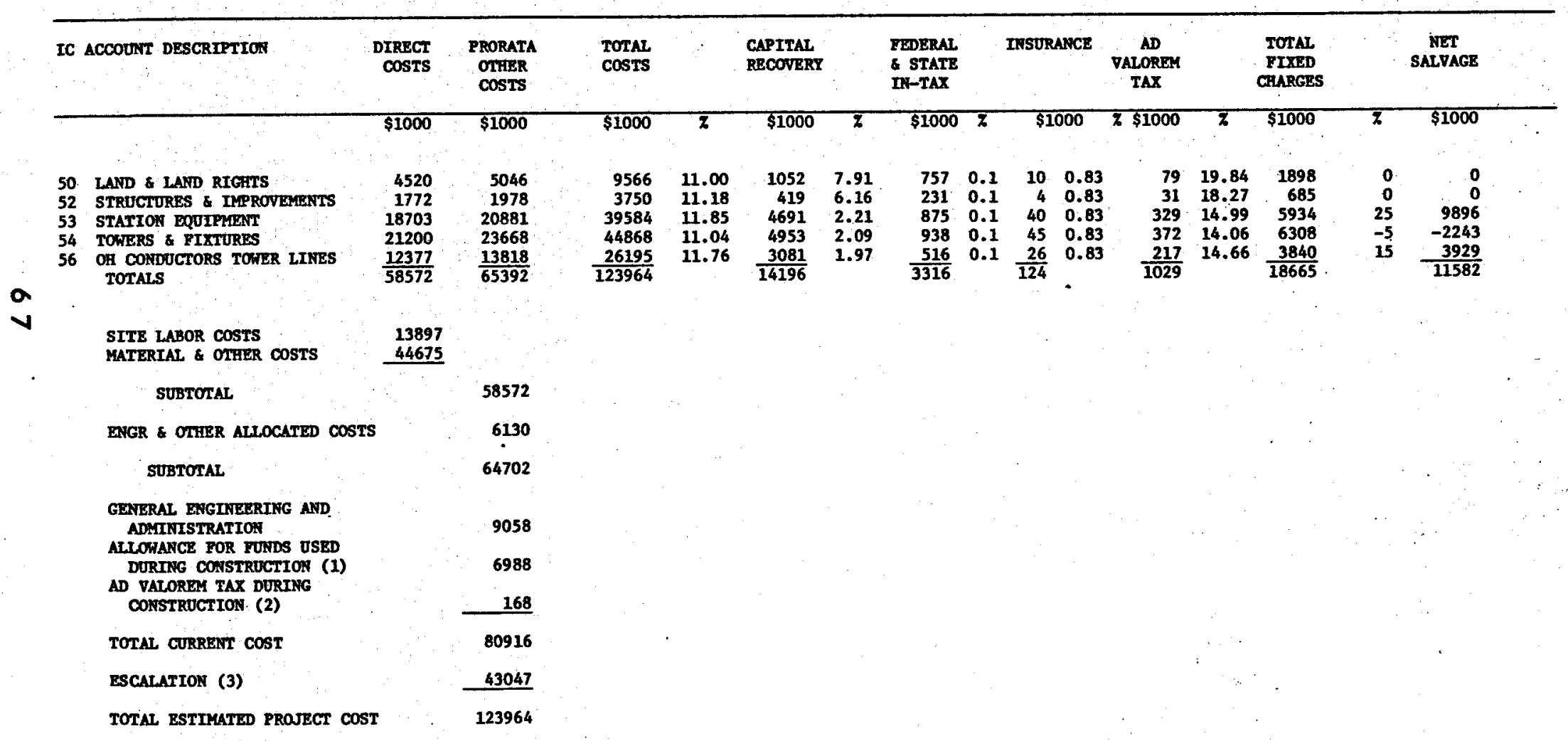




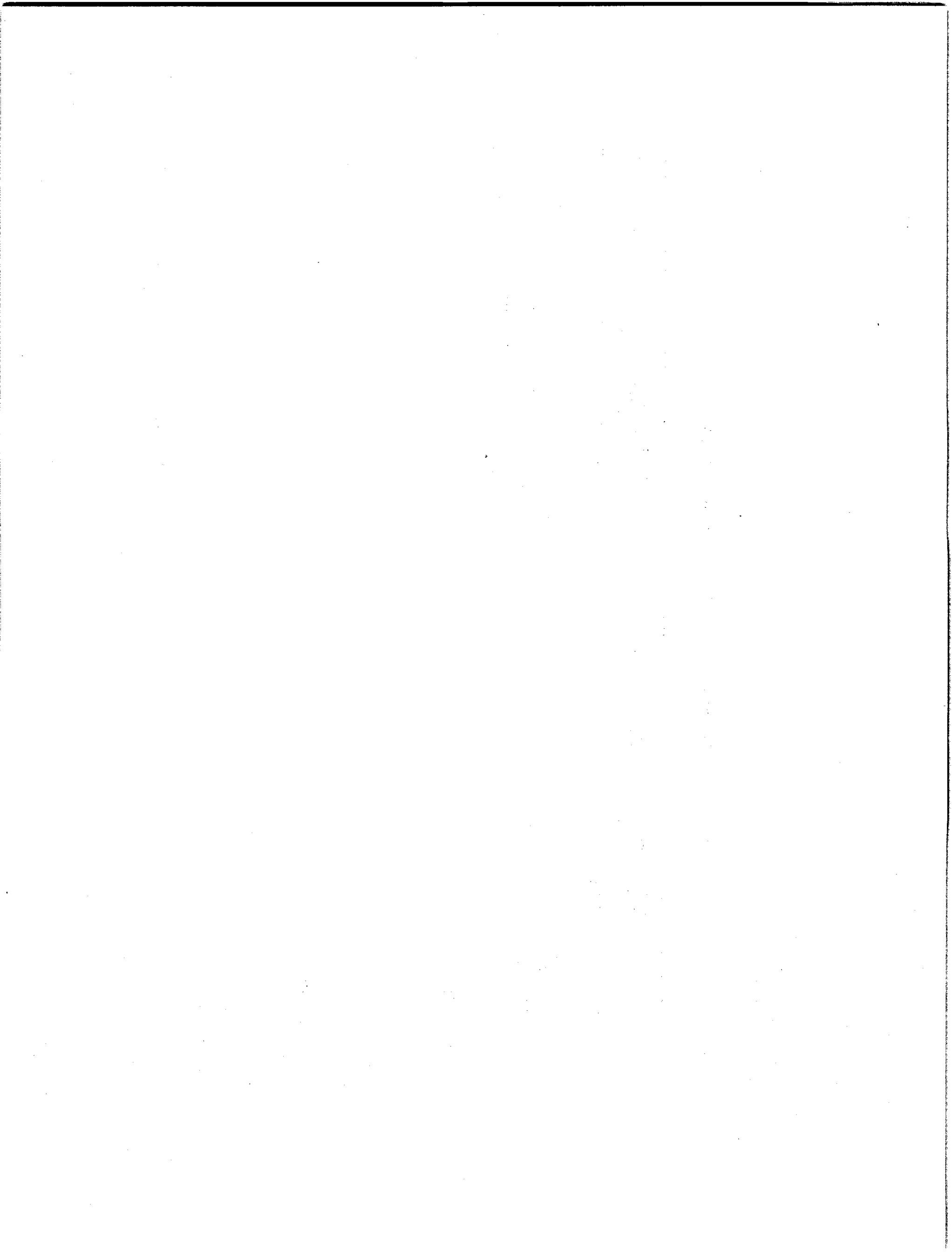


ATTACHMENT C

AN ANALYSIS OF RR AND DCF APPROACHES 
AN ANALYSIS OF RR AND DCF APPROACHES

The following symbols will be used:

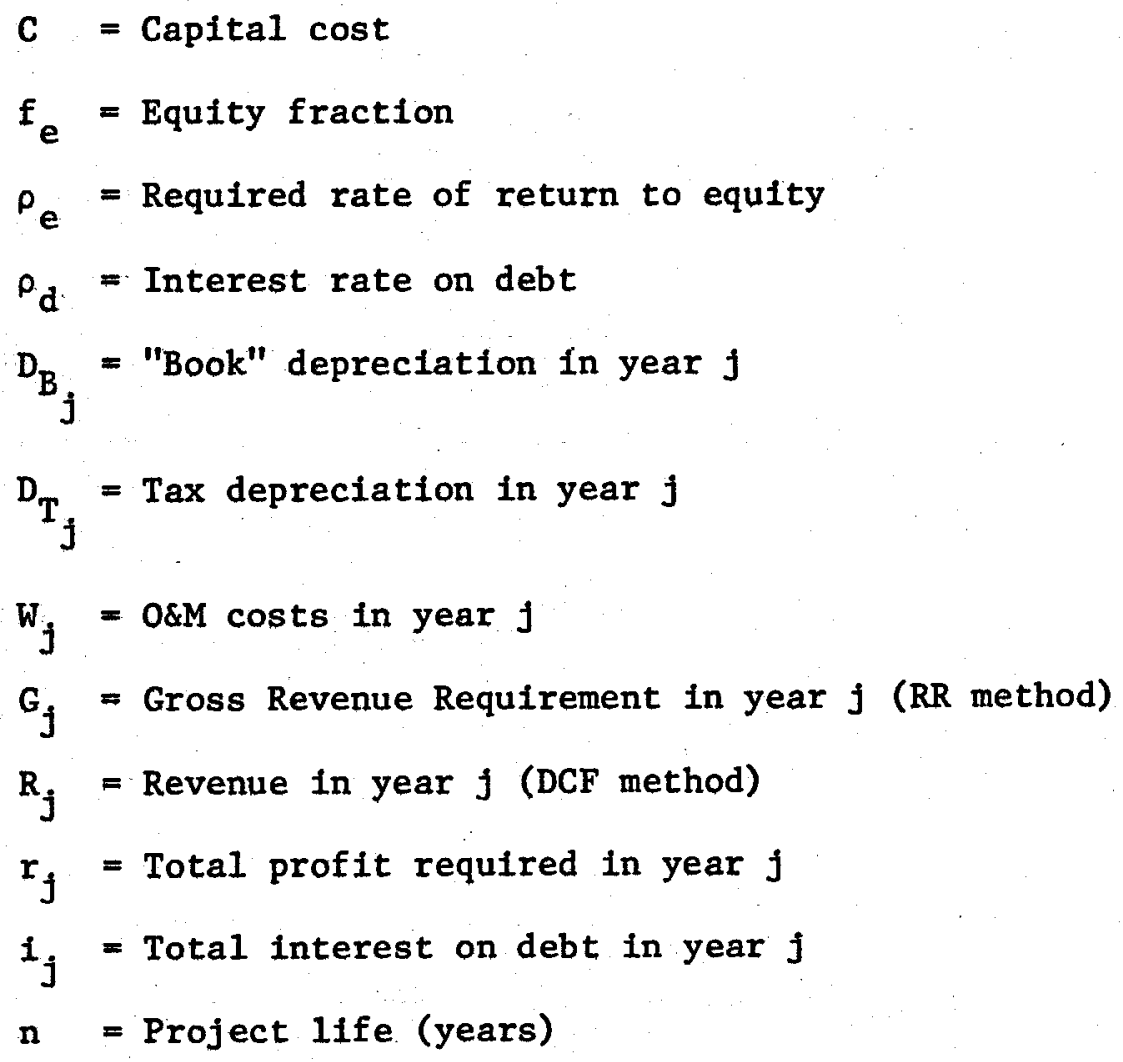

For the RR method, income tax llability in year $j$ is: $\operatorname{Tax}_{j}=t\left(G_{j}-D_{T_{j}}-W_{j}-I_{j}\right)$ where $t=$ tax rate and the required profit is:

$$
r_{j}=G_{j}-D_{B_{j}}-W_{j}-i_{j}-\operatorname{Tax}_{j}
$$

assuming that negative tax liabilities can be counted as income.

So that

$$
G_{j}=W_{j}+I_{j}+\frac{1}{1-t}\left(r_{j}+D_{B_{j}}-t D_{r_{j}}\right)
$$


Now, the unleveraged cash flow in year $j$ is:

$$
\begin{aligned}
C F_{j} & =R_{j}-W_{j}-\operatorname{Tax}_{j} \\
& =R_{j}-W_{j}-t\left(R_{j}-D_{r_{j}}-W_{j}-1_{j}\right) \\
& =(1-t) R_{j}-(1-t) W_{j}+t D_{T_{j}}+t 1_{j}
\end{aligned}
$$

Let $P_{d}()$ be the present-value operator, where $P_{d}\left(x_{j}\right)=\sum_{d=1}^{n} x_{j} d^{j}$ where $d$ is some discount rate. Then by definition, $R_{j}$ is chosen so that, for a given $d$, from equation (2):

$$
C=(i-t) P_{d}\left(R_{j}\right)-(1-t) P_{d}\left(W_{f}\right)+t P_{d}\left(D_{T_{j}}\right)+t P_{d}\left(1_{f}\right)
$$

Dropping the subscript $d$, we obtain:

$$
P\left(R_{j}\right)=\frac{C}{1-t}+P\left(W_{j}\right)-\frac{t}{1-t}\left(P\left(D_{T_{j}}\right)+P\left(C_{j}\right)\right)
$$

For the RR method, $G_{f}$ is chosen so that, from eqn (1):

$P\left(G_{j}\right)=P\left(W_{j}\right)+P\left(I_{j}\right)+\frac{1}{(1-t)}\left(P\left(r_{f}\right)+P\left(D_{B_{j}}\right)-t P\left(D_{T_{j}}\right)\right)$

$$
\text { If } P\left(R_{j}\right)=P\left(G_{j}\right) \text {, from equations (3) and (4): }
$$

$$
\begin{aligned}
& \frac{C}{(1-t)}+P\left(W_{j}\right)-\frac{t}{(1-t)} P\left(D_{T_{j}}\right)-\frac{t}{(1-t)} P\left(i_{j}\right)=P\left(W_{j}\right)+P\left(i_{j}\right)+P\left(V_{j}\right) \\
&+\frac{1}{(1-t)} P\left(D_{B_{j}}\right)-\frac{t}{(1-t)} P\left(D_{T_{j}}\right) \\
& \therefore \quad C=P\left(1_{j}\right)+P\left(r_{j}\right)+P\left(D_{B_{j}}\right)
\end{aligned}
$$


Assuming that $D_{B_{j}}=1 / n$, and that debt and equity are retired at the same uniform rate, then:

$$
\begin{aligned}
& c=C \sum_{j=1}^{n} d^{j}\left(\frac{(n+1-j)}{n} \cdot\left(1-f_{e}\right) \rho_{d}+\frac{(n+1-j)}{n} f_{e} e_{e}+\frac{1}{n}\right) \\
& \therefore n=d\left(\frac{1-d^{n}}{1-d}\right)+\left[\left(1-f_{e}\right) \rho_{d}+f_{e} \rho_{e}\right] \frac{d}{(1-d)^{2}}\left[n-(n+1) d+d^{n+1}\right]
\end{aligned}
$$

Write $\left(1-f_{e}\right) \rho_{d}+f_{e} \rho_{e}=r$. Then

$$
n=\frac{d}{(1-d)}\left(1-d^{n}\right)+\frac{d r}{(1-d)^{2}}\left[n-(n+1) d+d^{n+1}\right]
$$

Solving for $r$ yields

$$
\begin{gathered}
r=\frac{1}{d}\left[\frac{n-(2 n+1) d+(n+1) d^{2}+d^{n+1}-d^{n+1}}{n-(n+1) d+d^{n+1}}\right]=\frac{1-d}{d} \\
\quad \therefore d=\frac{1}{1+r}
\end{gathered}
$$

This Indicates that the revenue streams derived by the DCF and RR methods are equivalent if the tax-free discount rate is used*.

* A more detailed treatment of the situation may be found in Wong, Y.C.: "The Discounted Cash Flow (DCF) and Revenue Requirements (RR) Methodologies in Energy Cost Analysis", Institute for Energy Analysis, Oak Ridge Assoclated Universities, Oak Ridge, Tennessee 37830. 